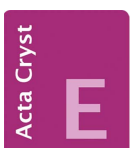

CRYSTALLOGRAPHIC COMMUNICATIONS

ISSN 2056-9890

Received 25 August 2021

Accepted 11 October 2021

Edited by J. Ellena, Universidade de Sâo Paulo, Brazil

Keywords: Nickel; $\alpha$-aminooxime; $(R)$-limonene; crystal structure.

CCDC reference: 2115017

Supporting information: this article has supporting information at journals.iucr.org/e

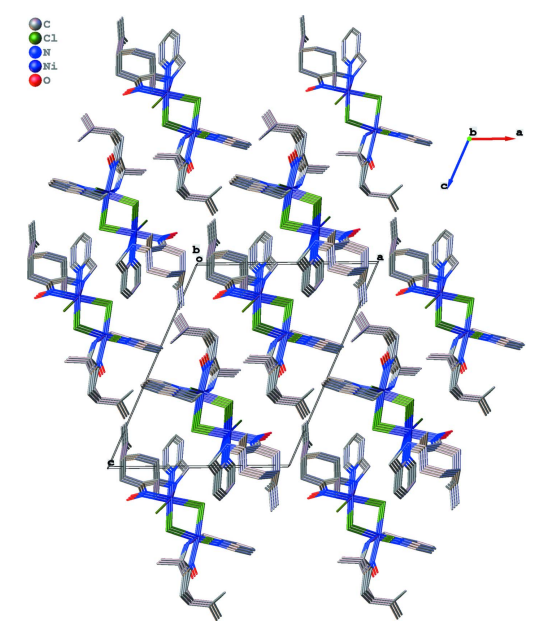

OPEN $\odot$ ACCESS

\section{Synthesis and crystal structure of a new chiral $a$-aminooxime nickel(II) complex}

\author{
Yasmina Homrani, ${ }^{a}$ Abdelaziz Dahdouh, ${ }^{a}$ Mohamed Amin El Amrani, ${ }^{a}$ Pauline \\ Loxq, ${ }^{b}$ Frédéric Capet, ${ }^{b}$ Isabelle Suisse ${ }^{b}$ and Mathieu Sauthier ${ }^{\text {b* }}$
}

\begin{abstract}
aLaboratoire de Chimie Organique Appliquée, Faculté des Sciences, BP 2121, Université Abdelmalek Essaadi, Tétouan, Morocco, and ${ }^{\mathbf{b}}$ Univ. Lille, CNRS, Centrale Lille, Univ. Artois, UMR 8181, UCCS, Unité de Catalyse et Chimie du Solide, F-59000, Lille, France. *Correspondence e-mail: mathieu.sauthier@univ-lille.fr
\end{abstract}

A dinuclear nickel complex with $(S)$-limonene based aminooxime ligand has been isolated and its crystal structure determined. The resolved structure of dichloridobis $\{(2 S, 5 R)$-2-methyl-5-(prop-1-en-2-yl)-2-[(pyridin-2-yl)methylamino]cyclohexan-1-one oxime\}dinickel(II), $\left[\mathrm{Ni}_{2} \mathrm{Cl}_{2}\left(\mathrm{C}_{16} \mathrm{H}_{23} \mathrm{ClN}{ }_{3} \mathrm{O}\right)_{2}\right]$, at $100 \mathrm{~K}$ has monoclinic $\left(P 2_{1}\right)$ symmetry. The two $\mathrm{Ni}^{\mathrm{II}}$ ions in the dinuclear complex are each coordinated in a distorted octahedral environment by three nitrogen atoms, a terminal chloride and two $\mu$ bridging chlorides. Each oxime ligand is coordinated to nickel(II) by the three nitrogen atoms, leading to two fivemembered chelate rings, each displaying an envelope conformation. In the crystal, numerous intermolecular and intramolecular hydrogen bonds lead to the formation of a three-dimensional network structure.

\section{Chemical context}

Asymmetric synthesis allows the preparation of enantiomerically enriched compounds either by using a chiral auxiliary, which will be temporarily introduced, or by using catalytic procedures (Gawley \& Aubé, 2012). This latter method is particularly attractive as it contributes to the development of green chemistry, which maximizes efficiency and minimizes hazardous effects on human health and the environment (Anastas \& Zimmerman, 2013). Thus, asymmetric catalysis avoids synthetic steps and only catalytic amounts of the optically pure auxiliary are needed (Ojima, 2010). As part of the development of this chemistry, the synthesis of new chiral organometallic complexes is always challenging. The pivotal point is then the synthesis of optically pure ligands, which will be coordinated to the metal center. In terms of sustainable chemistry, using the chiral pool to develop new ligands is most interesting (Elalami et al., 2015). Coordination metal complexes containing terpenoid fragments are widely used in the pharmaceutical field and in catalysis. We have therefore developed ligands based on terpenes such as pinene and limonene (El Alami et al., 2009, 2015; Chahboun et al., 2012). In particular, the synthesis of optically pure aminooxime ligands has been performed successfully from $(R)$ limonene (El Alami et al., 2012). These compounds possess structures with two or three nitrogen atoms as donor heteroatoms that could coordinate to the metal center. They have advantageously replaced phosphine ligands, which are generally unstable under air. Ruthenium (Benabdelouahab et al., 2015) and palladium (de la Cueva-Alique et al., 2019) 
complexes have already been synthezised with these ligands. Here we report the first synthesis of a limonene-based $\alpha$ aminooxime nickel complex and its crystal structure. In the dinuclear title complex, each nickel ion is coordinated by (1S,4R)-1-picolylamino- $p$-menth-8-en-2-one oxime. The ligand was first synthesized from $(R)$-limonene through the addition of nitrosyl chloride, $\mathrm{NOCl}$, to a picolylamine moiety, allowing the formation of the oxime moiety.

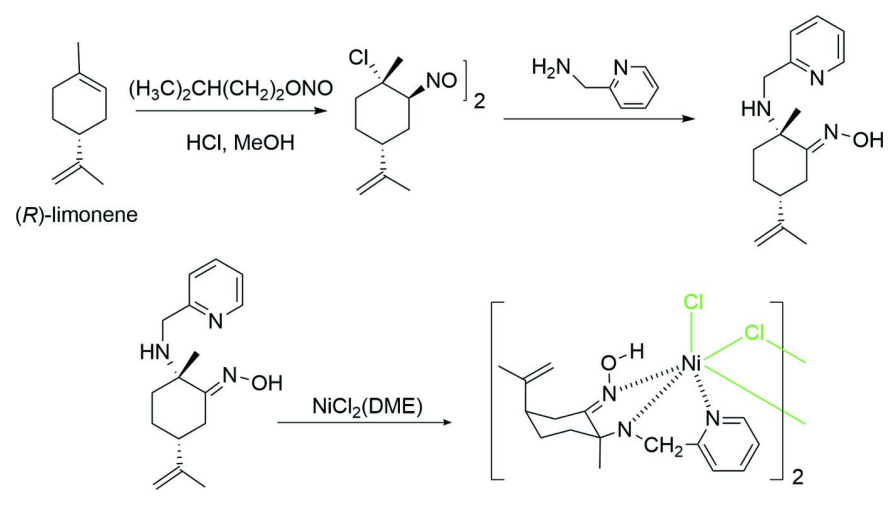

\section{Structural commentary}

The title compound (Fig. 1) crystallizes in the monoclinic space group $P 2_{1}$ with two chiral molecules per unit cell. The two $\mathrm{Ni}^{\mathrm{II}}$ ions in the dinuclear complex are each coordinated by three nitrogen atoms, a terminal chloride and two $\mu$ bridging chlorides. The environment around each metal center can then be described as a distorted octahedron with N1-Ni1 - N2 and $\mathrm{Cl} 1-\mathrm{Ni1}-\mathrm{Cl} 3$ angles of 79.91 (13) and $91.99(4)^{\circ}$, respectively, together with $\mathrm{Cl} 1-\mathrm{Ni} 1-\mathrm{N} 2$ and $\mathrm{Cl} 2-\mathrm{Ni1}-\mathrm{N} 1$ angles of $165.04(11)$ and $88.69(10)^{\circ}$, respectively. A similar arrangement can be found around the Ni2 atom [N4-Ni2$\mathrm{N} 5, \mathrm{Cl} 2-\mathrm{Ni} 2-\mathrm{Cl} 4, \mathrm{Cl} 4-\mathrm{Ni} 2-\mathrm{N} 5$ and $\mathrm{Cl} 4-\mathrm{Ni} 2-\mathrm{N} 4=$ 79.7 (2), 99.38 (4), 166.04 (12) and $93.24(16)^{\circ}$, respectively].

Each aminooxime ligand is coordinated to nickel(II) by the three nitrogen atoms, leading to two five-membered chelate rings, each displaying an envelope conformation (with $\mathrm{N} 2$ as the flap for $\mathrm{Ni1} / \mathrm{N} 1 / \mathrm{C} 5 / \mathrm{C} 6 / \mathrm{N} 2$ and $\mathrm{N} 5$ for $\mathrm{Ni2} / \mathrm{N} 4 / \mathrm{C} 21 / \mathrm{C} 22 /$ N5). The six-membered carbocycles of the limonene units adopt a chair conformation. The lengths of the Ni1-N1, $\mathrm{Ni1}-\mathrm{N} 2$ and Ni1-N3 bonds are 2.077 (3), $2.126(4)$ and

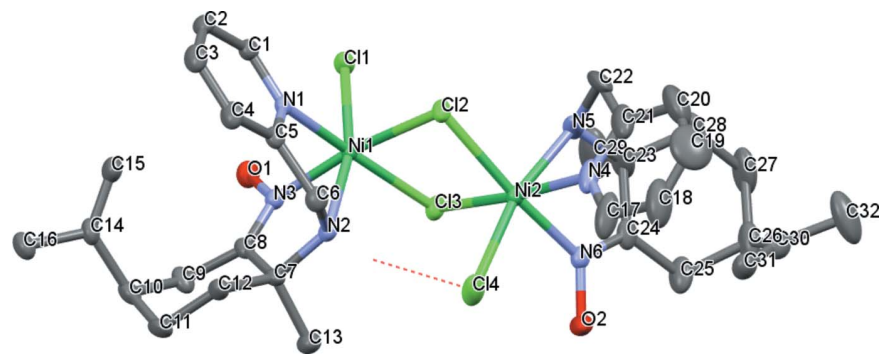

Figure 1

Displacement ellipsoid plot at the $50 \%$ probability level for $\mathrm{Ni}_{2}$ (aminooxime) ${ }_{2} \mathrm{Cl}_{4} . \mathrm{H}$ atoms are omitted for clarity.
Table 1

Hydrogen-bond geometry $\left(\AA,^{\circ}\right)$.

\begin{tabular}{lllll}
\hline$D-\mathrm{H} \cdots A$ & $D-\mathrm{H}$ & $\mathrm{H} \cdots A$ & $D \cdots A$ & $D-\mathrm{H} \cdots A$ \\
\hline $\mathrm{O} 1-\mathrm{H} 1 \cdots \mathrm{Cl} 1$ & $0.85(7)$ & $2.32(6)$ & $3.009(4)$ & $139(6)$ \\
$\mathrm{N} 2-\mathrm{H} 2 \cdots \mathrm{Cl} 4$ & $0.77(5)$ & $2.46(5)$ & $3.209(4)$ & $166(5)$ \\
$\mathrm{O} 2-\mathrm{H} 2 A \cdots \mathrm{Cl} 4$ & $0.76(8)$ & $2.31(7)$ & $2.978(4)$ & $147(7)$ \\
$\mathrm{C} 3-\mathrm{H} 3 \cdots \mathrm{O} 1^{\mathrm{i}}$ & 0.95 & 2.58 & $3.432(5)$ & 149 \\
$\mathrm{C} 1-\mathrm{H} 1 A \cdots \mathrm{Cl} 1$ & 0.95 & 2.75 & $3.369(5)$ & 124 \\
$\mathrm{C} 6-\mathrm{H} 6 A \cdots \mathrm{Cl} 2$ & 0.99 & 2.76 & $3.309(5)$ & 115 \\
$\mathrm{C} 11-\mathrm{H} 11 B \cdots \mathrm{Cl} 3^{\mathrm{ii}}$ & 0.99 & 2.64 & $3.573(5)$ & 156 \\
$\mathrm{C} 17-\mathrm{H} 17 \cdots \mathrm{Cl} 4$ & 0.95 & 2.69 & $3.327(6)$ & 125 \\
$\mathrm{C} 26-\mathrm{H} 26 \cdots \mathrm{O} 2^{\mathrm{iii}}$ & 1.00 & 2.56 & $3.489(6)$ & 154 \\
$\mathrm{C} 22-\mathrm{H} 22 B \cdots \mathrm{Cl} 2$ & 0.99 & 2.81 & $3.352(6)$ & 115 \\
$\mathrm{C} 19-\mathrm{H} 19 \cdots \mathrm{Cl} 1^{\text {iv }}$ & 0.95 & 2.64 & $3.570(7)$ & 167 \\
\hline
\end{tabular}

Symmetry codes: (i) $-x+2, y-\frac{1}{2},-z+1$; (ii) $-x+1, y-\frac{1}{2},-z+1$; (iii) $-x, y+\frac{1}{2},-z$; (iv) $-x+1, y-\frac{1}{2},-z$.

2.041 (3) $\AA$, respectively, while Ni2-N4, Ni2-N5 and Ni2N6 are 2.095 (4), 2.103 (4) and 2.027 (3) ̊. Atoms Cl1 and Cl4 are in a trans-position at distances of 2.4408 (12) and 2.4077 (14) $\AA$ from the metal centers Ni1 and Ni2, respectively. The two metal centers are linked by two bridging $\mathrm{Cl}$ atoms with an average $\mathrm{Ni}-\mathrm{Cl}$ distance of $2.42 \AA$, which is normal for these bond lengths. All these values compare well with literature values. The two nickel ions are separated by a distance of 3.5198 (7) $\AA$, which is similar to average values (Zheng et al., 2010; Cheng et al., 2012).

\section{Supramolecular features}

The crystal structure is stabilized by numerous intermolecular and intramolecular hydrogen bonds (Table 1), which link the component into a three-dimensional network (Figs. 2 and 3).

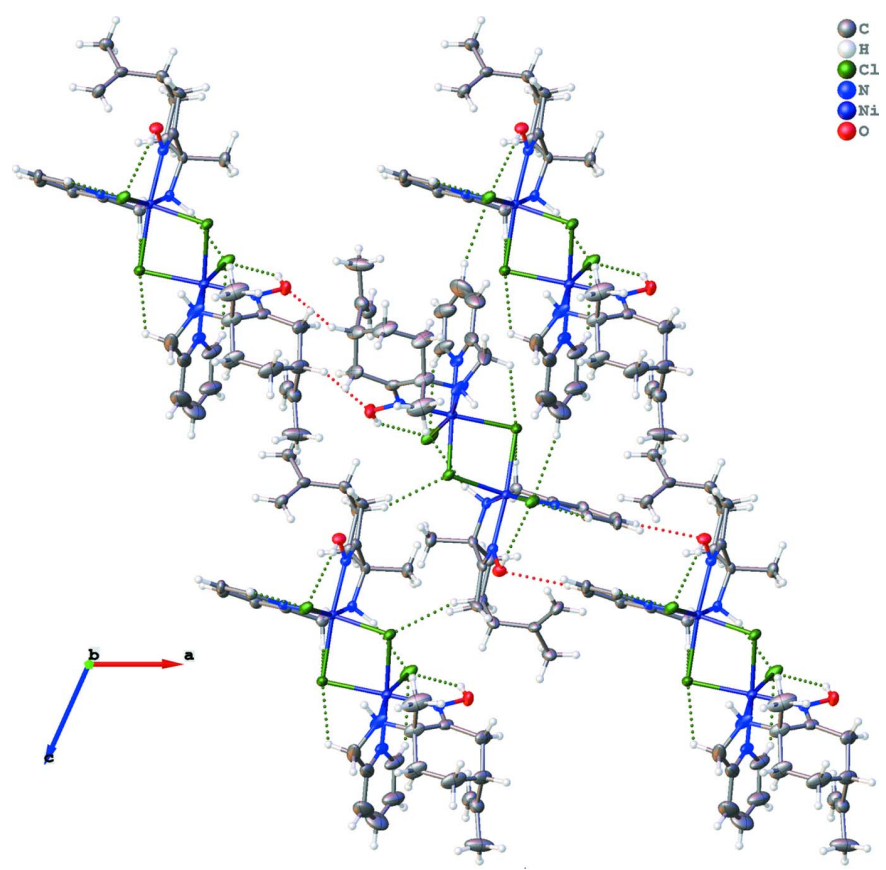

Figure 2

Intermolecular and intramolecular hydrogen bonds in the structure, shown as dashed lines. 


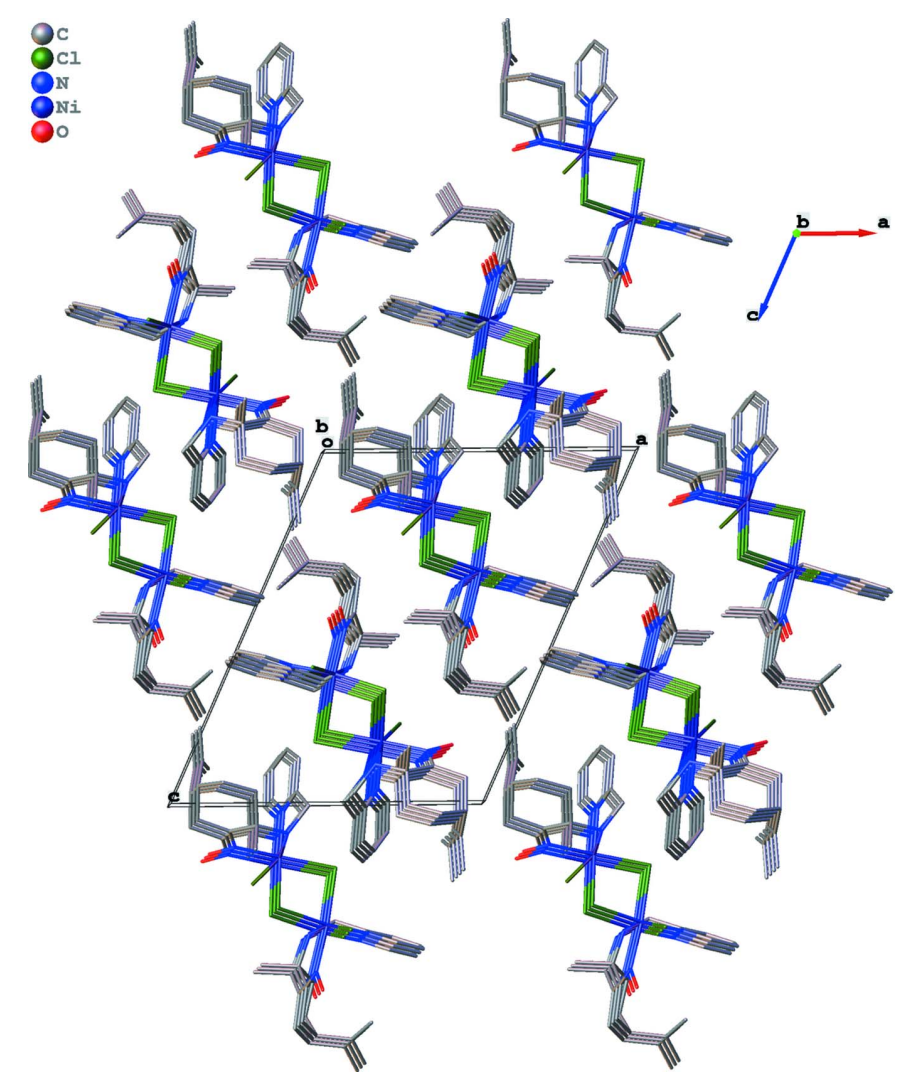

Figure 3

Packing diagram.

In particular, the two $\{\mathrm{Ni}($ aminoxime $) \mu-\mathrm{Cl}\} \mathrm{Cl}$ units are slightly asymmetrical with the existence of a hydrogenbonding interaction between the amine $\mathrm{N} 2-\mathrm{H} 2$ linked to Ni1 and the chlorine atom $\mathrm{Cl} 4$ linked to $\mathrm{Ni} 2$. In addition, the two oxygen atoms $\mathrm{O} 1$ and $\mathrm{O} 2$ of the oxime groups are involved in intramolecular $\mathrm{O} 1-\mathrm{H} 1 \cdots \mathrm{Cl} 1$ and $\mathrm{O} 2-\mathrm{H} 2 A \cdots \mathrm{Cl} 4$ hydrogen bonds and in intermolecular $\mathrm{C} 3-\mathrm{H} 3 \cdots \mathrm{O} 1$ and $\mathrm{C} 26-$ $\mathrm{H} 26 \cdots \mathrm{O} 2$ interactions.

\section{Database survey}

The aminooxime ligand used in this study was previously reacted with palladium and platinum precursors, generating three $N$-coordinated cationic complexes as enantiopure compounds (de la Cueva-Alique et al., 2019). A heteronuclear $\mathrm{Ti}^{\mathrm{IV}} / \mathrm{Pd}^{\mathrm{II}}$ complex has also been described. The compounds were studied to assess their potential biological activity, a high anticancer activity (de la Cueva-Alique et al., 2019).

\section{Synthesis and crystallization}

To a solution of $\mathrm{Ni}^{\mathrm{II}}$ chloride ethylene glycol dimethyl ether $(0.15 \mathrm{~g}, 1.48 \mathrm{mmol})$ in $\mathrm{MeOH}(5 \mathrm{~mL})$ was added $(1 S, 4 R)-1$ picolylamino- $p$-menth-8-en-2-one-oxime $(0.101 \mathrm{~g}, 0.36 \mathrm{mmol})$ dissolved in $\mathrm{MeOH}(3 \mathrm{~mL})$. The solution turned green. The mixture was stirred overnight at room temperature during which time the mixture changed color to blue-green. The
Table 2

Experimental details.

\begin{tabular}{|c|c|}
\hline \multicolumn{2}{|l|}{ Crystal data } \\
\hline Chemical formula & {$\left[\mathrm{Ni}_{2} \mathrm{Cl}_{2}\left(\mathrm{C}_{16} \mathrm{H}_{23} \mathrm{ClN}_{3} \mathrm{O}\right)_{2}\right]$} \\
\hline$M_{\mathrm{r}}$ & 805.97 \\
\hline Crystal system, space group & Monoclinic, $P 2_{1}$ \\
\hline Temperature $(\mathrm{K})$ & 100 \\
\hline$a, b, c(\AA)$ & $\begin{array}{l}13.3729(9), 8.9363(7) \\
16.4248(16)\end{array}$ \\
\hline$\beta\left(^{\circ}\right)$ & $114.014(2)$ \\
\hline$V\left(\AA^{6}\right)$ & $1792.9(3)$ \\
\hline$Z$ & 2 \\
\hline Radiation type & Mo $K \alpha$ \\
\hline$\mu\left(\mathrm{mm}^{-1}\right)$ & 1.39 \\
\hline Crystal size $(\mathrm{mm})$ & $0.21 \times 0.17 \times 0.12$ \\
\hline \multicolumn{2}{|l|}{ Data collection } \\
\hline Diffractometer & Bruker APEXII CCD \\
\hline Absorption correction & $\begin{array}{l}\text { Multi-scan (SADABS; Krause et } \\
\quad \text { al., 2015) }\end{array}$ \\
\hline$T_{\min }, T_{\max }$ & $0.669,0.746$ \\
\hline $\begin{array}{l}\text { No. of measured, independent and } \\
\text { observed }[I>2 \sigma(I)] \text { reflections }\end{array}$ & $42747,10769,9436$ \\
\hline$R_{\text {int }}$ & 0.037 \\
\hline$(\sin \theta / \lambda)_{\max }\left(\AA^{-1}\right)$ & 0.714 \\
\hline \multicolumn{2}{|l|}{ Refinement } \\
\hline$R\left[F^{2}>2 \sigma\left(F^{2}\right)\right], w R\left(F^{2}\right), S$ & $0.043,0.109,1.05$ \\
\hline No. of reflections & 10769 \\
\hline No. of parameters & 431 \\
\hline No. of restraints & 13 \\
\hline $\mathrm{H}$-atom treatment & $\begin{array}{l}\mathrm{H} \text { atoms treated by a mixture of } \\
\text { independent and constrained } \\
\text { refinement }\end{array}$ \\
\hline$\Delta \rho_{\max }, \Delta \rho_{\min }\left(\mathrm{e} \AA^{-3}\right)$ & $1.50,-1.19$ \\
\hline Absolute structure & $\begin{array}{l}\text { Flack } x \text { determined using } 3850 \\
\text { quotients }\left[\left(I^{+}\right)-\left(I^{-}\right)\right] /\left[\left(I^{+}\right)+\left(I^{-}\right)\right] \\
\quad \text { (Parsons } \text { et al. } 2013)\end{array}$ \\
\hline Absolute structure parameter & -0.009 (4) \\
\hline
\end{tabular}

Computer programs: APEX2 and SAINT (Bruker, 2019), SHELXT (Sheldrick, 2015a), SHELXL (Sheldrick, 2015b) and OLEX2 (Dolomanov et al., 2009).

solvent was then evaporated to produce a crude solid that was washed with diethyl ether before crystallization. Single crystals were grown by slow diffusion at room temperature of diethyl ether into a dichloromethane solution. Elemental analysis calculated for $\mathrm{C}_{32} \mathrm{H}_{46} \mathrm{Cl}_{4} \mathrm{~N}_{6} \mathrm{Ni}_{2} \mathrm{O}_{2}: \mathrm{C}, 46.33 ; \mathrm{H}, 5.54 ; \mathrm{N}$, 9.65. Found: C, 46.35; H, 5.672; N, 9.77.

\section{Refinement}

Crystal data, data collection and structure refinement details are summarized in Table $2 . \mathrm{N}$ - and $\mathrm{O}$-bound atoms were refined with the restraint $U_{\text {iso }}(\mathrm{H})=1.2 U_{\text {eq }}(\mathrm{N})$ or $1.5 U_{\text {eq }}(\mathrm{O})$. H

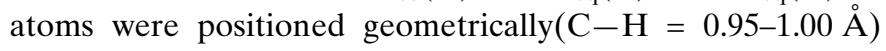
and refined as riding with $U_{\text {iso }}(\mathrm{H})=1.2 U_{\text {eq }}(\mathrm{C})$ or $1.5 U_{\text {eq }}(\mathrm{C}-$ methyl)

\section{Acknowledgements}

We would like to thank Céline Delabre for the elemental analysis. 


\section{Funding information}

The authors thank the Ministère de l'Enseignement Supérieur de la Recherche et de l'Innovation (France) and the Ministère de la Recherche (Morocco) for financial support. The Chevreul Institute (FR 2638), Ministry of Higher Education, Research and Innovation, Région Hauts de France and FEDER are recognized for funding of X-ray diffractometers.

\section{References}

Anastas, P. T. \& Zimmerman, J. B. (2013). Environ. Sci. Technol. 37, 95A-101A.

Benabdelouahab, Y., Muñoz-Moreno, L., Frik, M., de la CuevaAlique, I., El Amrani, M. A., Contel, M., Bajo, A. M., Cuenca, T. \& Royo, E. (2015). Eur. J. Inorg. Chem. pp. 2295-2307.

Bruker (2019). APEX2 and SAINT. Bruker AXS Inc., Madison Wisconsin, USA.

Chahboun, G., Brito, J. A., Royo, B., El Amrani, M. A., GómezBengoa, E., Mosquera, M. E. G., Cuenca, T. \& Royo, E. (2012). Eur. J. Inorg. Chem. pp. 2940-2949.

Cheng, T.-P., Liao, B.-S., Liu, Y.-H., Peng, S.-M. \& Liu, S.-T. (2012). Dalton Trans. 41, 3468-3473.
Cueva-Alique, I. de la, Muñoz-Moreno, L., de la Torre-Rubio, E., Bajo, A. M., Gude, L., Cuenca, T. \& Royo, E. (2019). Dalton Trans. 48, 14279-14293.

Dolomanov, O. V., Bourhis, L. J., Gildea, R. J., Howard, J. A. K. \& Puschmann, H. (2009). J. Appl. Cryst. 42, 339-341.

Elalami, M. S., Dahdouh, A. A., Mansour, A. I., ElAmrani, M. A., Suisse, I., Mortreux, A. \& Agbossou-Niedercorn, F. (2009). C. $R$. Chim. 12, 1253-1258.

El Alami, M. S. I., El Amrani, M. A., Agbossou-Niedercorn, F., Suisse, I. \& Mortreux, A. (2015). Chem. Eur. J. 21, 1398-1413.

Gawley, R. E. \& Aubé, J. (2012). Principles and applications of asymmetric synthesis, 2nd ed. Amsterdam: Elsevier Science

El Alami, M. S. I., El Amrani, M. A., Dahdouh, A., Roussel, P., Suisse, I. \& Mortreux, A. (2012). Chirality, 24, 675-682.

Krause, L., Herbst-Irmer, R., Sheldrick, G. M. \& Stalke, D. (2015). J. Appl. Cryst. 48, 3-10.

Ojima, I. (2010). Catalytic asymmetric synthesis, 3rd ed. Hoboken: Wiley

Parsons, S., Flack, H. D. \& Wagner, T. (2013). Acta Cryst. B69, 249259.

Sheldrick, G. M. (2015a). Acta Cryst. A71, 3-8.

Sheldrick, G. M. (2015b). Acta Cryst. C71, 3-8.

Zheng, L., Zhang, S., Li, K., Chen, W., Chen, Y., Xu, B., Hu, B., Li, Y. \& Li, W. (2010). J. Mol. Struct. 984, 153-156. 


\section{supporting information}

Acta Cryst. (2021). E77, 1116-1119 [https://doi.org/10.1107/S2056989021010537]

Synthesis and crystal structure of a new chiral $\alpha$-aminooxime nickel(II) complex

Yasmina Homrani, Abdelaziz Dahdouh, Mohamed Amin El Amrani, Pauline Loxq, Frédéric

Capet, Isabelle Suisse and Mathieu Sauthier

Computing details

Data collection: APEX2 (Bruker, 2019); cell refinement: SAINT (Bruker, 2019); data reduction: SAINT(Bruker, 2019); program(s) used to solve structure: ShelXT (Sheldrick, 2015a); program(s) used to refine structure: SHELXL (Sheldrick, 2015b); molecular graphics: OLEX2 (Dolomanov et al., 2009); software used to prepare material for publication: OLEX2 (Dolomanov et al., 2009).

Dichloridobis\{(2S,5R)-2-methyl-5-(prop-1-en-2-yl)-2-[(pyridin-2-yl)methylamino]cyclohexan-1-one oxime\}dinickel(II)

Crystal data

$\left[\mathrm{Ni}_{2} \mathrm{Cl}_{2}\left(\mathrm{C}_{16} \mathrm{H}_{23} \mathrm{ClN}_{3} \mathrm{O}\right)_{2}\right]$

$M_{r}=805.97$

Monoclinic, $P 2_{1}$

$a=13.3729(9) \AA$

$b=8.9363(7) \AA$

$c=16.4248(16) \AA$

$\beta=114.014(2)^{\circ}$

$V=1792.9(3) \AA^{3}$

$Z=2$

Data collection

Bruker APEXII CCD diffractometer

Radiation source: microfocus sealed X-ray tube $\varphi$ and $\omega$ scans

Absorption correction: multi-scan

(SADABS; Krause et al., 2015)

$T_{\min }=0.669, T_{\max }=0.746$

42747 measured reflections

Refinement

Refinement on $F^{2}$

Least-squares matrix: full

$R\left[F^{2}>2 \sigma\left(F^{2}\right)\right]=0.043$

$w R\left(F^{2}\right)=0.109$

$S=1.05$

10769 reflections

431 parameters

13 restraints

Primary atom site location: dual
$F(000)=840$

$D_{\mathrm{x}}=1.493 \mathrm{Mg} \mathrm{m}^{-3}$

Mo $K \alpha$ radiation, $\lambda=0.71073 \AA$

Cell parameters from 9996 reflections

$\theta=2.7-30.0^{\circ}$

$\mu=1.39 \mathrm{~mm}^{-1}$

$T=100 \mathrm{~K}$

Block, green

$0.21 \times 0.17 \times 0.12 \mathrm{~mm}$

10769 independent reflections

9436 reflections with $I>2 \sigma(I)$

$R_{\text {int }}=0.037$

$\theta_{\max }=30.5^{\circ}, \theta_{\min }=1.4^{\circ}$

$h=-17 \rightarrow 19$

$k=-12 \rightarrow 12$

$l=-23 \rightarrow 21$

Secondary atom site location: difference Fourier map

Hydrogen site location: mixed

$\mathrm{H}$ atoms treated by a mixture of independent and constrained refinement

$w=1 /\left[\sigma^{2}\left(F_{\mathrm{o}}^{2}\right)+(0.0581 P)^{2}+0.9636 P\right]$ where $P=\left(F_{\mathrm{o}}^{2}+2 F_{\mathrm{c}}^{2}\right) / 3$

$(\Delta / \sigma)_{\max }=0.001$

$\Delta \rho_{\max }=1.50 \mathrm{e} \AA^{-3}$ 
$\Delta \rho_{\min }=-1.18$ e $\AA^{-3}$
Absolute structure: Flack $x$ determined using 3850 quotients $\left[\left(I^{+}\right)-\left(I^{\prime}\right)\right] /\left[\left(I^{+}\right)+\left(I^{-}\right)\right]$(Parsons et al., 2013)

Absolute structure parameter: -0.009 (4)

Special details

Geometry. All esds (except the esd in the dihedral angle between two 1.s. planes) are estimated using the full covariance matrix. The cell esds are taken into account individually in the estimation of esds in distances, angles and torsion angles; correlations between esds in cell parameters are only used when they are defined by crystal symmetry. An approximate (isotropic) treatment of cell esds is used for estimating esds involving l.s. planes.

Fractional atomic coordinates and isotropic or equivalent isotropic displacement parameters $\left(\AA^{2}\right)$

\begin{tabular}{|c|c|c|c|c|}
\hline & $x$ & $y$ & $z$ & $U_{\text {iso }} * / U_{\text {eq }}$ \\
\hline Ni1 & $0.66455(4)$ & $0.50226(6)$ & $0.35327(3)$ & $0.01526(11)$ \\
\hline $\mathrm{Ni} 2$ & $0.41917(4)$ & $0.48960(6)$ & $0.15985(3)$ & $0.01813(12)$ \\
\hline $\mathrm{Cl} 2$ & $0.61155(8)$ & $0.45928(14)$ & $0.19208(7)$ & $0.0259(2)$ \\
\hline $\mathrm{Cl} 3$ & $0.48342(7)$ & $0.60270(12)$ & $0.30567(7)$ & $0.0212(2)$ \\
\hline $\mathrm{Cl1}$ & $0.74487(8)$ & $0.75170(12)$ & $0.37010(7)$ & $0.0249(2)$ \\
\hline $\mathrm{Cl} 4$ & $0.38501(9)$ & $0.25191(13)$ & $0.21270(9)$ & $0.0336(3)$ \\
\hline $\mathrm{O} 1$ & 0.7388 & $0.6301(4)$ & $0.5389(2)$ & $0.0244(7)$ \\
\hline H1 & $0.740(5)$ & $0.705(7)$ & $0.507(4)$ & $0.037^{*}$ \\
\hline N1 & $0.8111(3)$ & $0.3926(4)$ & $0.3786(2)$ & $0.0182(7)$ \\
\hline N3 & $0.6939(2)$ & $0.5043(5)$ & $0.4853(2)$ & $0.0183(6)$ \\
\hline $\mathrm{N} 2$ & $0.6216(3)$ & $0.2811(4)$ & $0.3745(2)$ & $0.0183(7)$ \\
\hline $\mathrm{H} 2$ & $0.563(4)$ & $0.289(6)$ & $0.339(3)$ & $0.022 *$ \\
\hline $\mathrm{O} 2$ & 0.1908 (3) & $0.4533(5)$ & $0.1497(2)$ & $0.0339(9)$ \\
\hline $\mathrm{H} 2 \mathrm{~A}$ & $0.226(6)$ & $0.389(9)$ & $0.176(5)$ & $0.051^{*}$ \\
\hline $\mathrm{C} 8$ & $0.6846(3)$ & $0.3877(5)$ & 0.5258 & $0.0203(8)$ \\
\hline $\mathrm{C} 5$ & 0.8008 & $0.2431(5)$ & 0.3699 & $0.0202(8)$ \\
\hline N6 & $0.2619(3)$ & $0.5471(4)$ & $0.1318(2)$ & $0.0212(8)$ \\
\hline N5 & $0.4081(3)$ & $0.6943(5)$ & 0.0936 & $0.0394(12)$ \\
\hline H5 & $0.468(5)$ & $0.736(8)$ & $0.140(4)$ & $0.047^{*}$ \\
\hline $\mathrm{C} 12$ & $0.7001(3)$ & $0.1123(6)$ & $0.5107(3)$ & $0.0250(9)$ \\
\hline $\mathrm{H} 12 \mathrm{~A}$ & 0.660298 & 0.022653 & 0.478112 & $0.030^{*}$ \\
\hline H12B & 0.770464 & 0.117488 & 0.504159 & $0.030^{*}$ \\
\hline $\mathrm{C} 14$ & $0.9033(3)$ & $0.2356(6)$ & 0.6758 & $0.0257(9)$ \\
\hline $\mathrm{C} 3$ & 0.9925 (4) & $0.2136(6)$ & $0.4131(3)$ & $0.0301(11)$ \\
\hline $\mathrm{H} 3$ & 1.054899 & 0.151943 & 0.425362 & $0.036^{*}$ \\
\hline $\mathrm{C} 9$ & $0.7192(4)$ & $0.3765(6)$ & $0.6253(3)$ & $0.0279(10)$ \\
\hline H9A & 0.653362 & 0.378843 & 0.638523 & $0.034 *$ \\
\hline H9B & 0.764996 & 0.464264 & 0.654444 & $0.034 *$ \\
\hline $\mathrm{C} 1$ & $0.9109(3)$ & $0.4531(5)$ & $0.4044(3)$ & $0.0220(9)$ \\
\hline H1A & 0.918464 & 0.558677 & 0.410777 & $0.026^{*}$ \\
\hline $\mathrm{C} 30$ & $0.0369(4)$ & $0.6416(7)$ & -0.1114 & $0.0357(12)$ \\
\hline N4 & $0.3669(3)$ & $0.4143(6)$ & $0.0282(3)$ & $0.0361(11)$ \\
\hline $\mathrm{C} 2$ & $1.0041(3)$ & $0.3660(6)$ & $0.4222(3)$ & $0.0277(10)$ \\
\hline $\mathrm{H} 2 \mathrm{~B}$ & 1.074096 & 0.411370 & 0.440161 & $0.033^{*}$ \\
\hline $\mathrm{C} 25$ & $0.1029(4)$ & $0.7072(7)$ & $0.0546(4)$ & $0.0384(13)$ \\
\hline
\end{tabular}




\begin{tabular}{|c|c|c|c|c|}
\hline $\mathrm{H} 25 \mathrm{~A}$ & 0.095090 & 0.786671 & 0.093629 & $0.046^{*}$ \\
\hline H25B & 0.059972 & 0.619493 & 0.058673 & $0.046 *$ \\
\hline $\mathrm{C} 7$ & $0.6314(3)$ & $0.2540(5)$ & 0.4678 & $0.0206(8)$ \\
\hline $\mathrm{C} 4$ & $0.8897(4)$ & $0.1499(6)$ & 0.3860 & $0.0278(10)$ \\
\hline H4 & 0.880183 & 0.044699 & 0.378533 & $0.033 *$ \\
\hline $\mathrm{C} 24$ & $0.2208(4)$ & $0.6641(5)$ & 0.0869 & $0.0258(10)$ \\
\hline C6 & 0.6855 & $0.1848(5)$ & $0.3402(3)$ & $0.0218(8)$ \\
\hline H6A & 0.649955 & 0.182503 & 0.274260 & $0.026^{*}$ \\
\hline H6B & 0.687313 & 0.081400 & 0.362311 & $0.026^{*}$ \\
\hline $\mathrm{C} 10$ & $0.7844(4)$ & $0.2319(6)$ & $0.6644(3)$ & $0.0281(10)$ \\
\hline H10 & 0.785669 & 0.219265 & 0.725250 & $0.034 *$ \\
\hline $\mathrm{C} 23$ & $0.2999(4)$ & $0.7724(5)$ & $0.0732(4)$ & $0.0365(12)$ \\
\hline $\mathrm{C} 15$ & $0.9419(4)$ & $0.3220(6)$ & $0.6296(3)$ & $0.0301(10)$ \\
\hline $\mathrm{H} 15 \mathrm{~A}$ & 1.016603 & 0.314817 & 0.638711 & $0.036^{*}$ \\
\hline H15B & 0.894916 & 0.391186 & 0.587469 & $0.036 *$ \\
\hline C31 & $0.0663(3)$ & $0.5006(7)$ & -0.0931 & $0.0322(10)$ \\
\hline H31A & 0.053020 & 0.430999 & -0.140077 & $0.039 *$ \\
\hline H31B & 0.100801 & 0.468485 & -0.032826 & $0.039 *$ \\
\hline C13 & 0.5139 & $0.2394(7)$ & 0.4628 & 0.0314 \\
\hline H13A & 0.471161 & 0.327732 & 0.433110 & $0.047 *$ \\
\hline H13B & 0.516636 & 0.231714 & 0.523147 & $0.047^{*}$ \\
\hline $\mathrm{H} 13 \mathrm{C}$ & 0.479378 & 0.149469 & 0.428806 & $0.047^{*}$ \\
\hline C11 & $0.7236(4)$ & $0.0951(6)$ & $0.6094(3)$ & $0.0306(11)$ \\
\hline H11A & 0.768517 & 0.004325 & 0.633040 & $0.037^{*}$ \\
\hline H11B & 0.653697 & 0.081821 & 0.615749 & $0.037 *$ \\
\hline C16 & $0.9776(4)$ & $0.1259(6)$ & $0.7427(3)$ & $0.0313(11)$ \\
\hline H16A & 0.980940 & 0.149758 & 0.802043 & $0.047 *$ \\
\hline H16B & 1.051172 & 0.132186 & 0.743621 & $0.047 *$ \\
\hline $\mathrm{H} 16 \mathrm{C}$ & 0.948965 & 0.024270 & 0.726130 & $0.047 *$ \\
\hline $\mathrm{C} 18$ & $0.2741(6)$ & $0.2441(10)$ & $-0.0937(4)$ & $0.0582(18)$ \\
\hline H18 & 0.234602 & 0.153747 & -0.115022 & $0.070 *$ \\
\hline $\mathrm{C} 17$ & $0.3151(5)$ & $0.2832(8)$ & $-0.0055(4)$ & $0.0493(16)$ \\
\hline H17 & 0.306660 & 0.213712 & 0.035033 & $0.059 *$ \\
\hline $\mathrm{C} 26$ & $0.0565(4)$ & $0.7638(7)$ & -0.0421 & $0.0423(14)$ \\
\hline $\mathrm{H} 26$ & -0.016160 & 0.810447 & -0.054167 & $0.051 *$ \\
\hline $\mathrm{C} 21$ & $0.3804(4)$ & $0.5139(10)$ & -0.0254 & $0.0504(17)$ \\
\hline $\mathrm{C} 28$ & $0.2479(5)$ & $0.8323(7)$ & $-0.0237(4)$ & $0.0486(16)$ \\
\hline $\mathrm{H} 28 \mathrm{~A}$ & 0.293285 & 0.915184 & -0.030027 & $0.058^{*}$ \\
\hline H28B & 0.247256 & 0.751483 & -0.065083 & $0.058^{*}$ \\
\hline C32 & $-0.0176(7)$ & $0.6926(9)$ & $-0.2067(4)$ & $0.068(2)$ \\
\hline $\mathrm{H} 32 \mathrm{~A}$ & 0.032890 & 0.756035 & -0.220891 & $0.101 *$ \\
\hline H32B & -0.083660 & 0.749858 & -0.215141 & $0.101 *$ \\
\hline $\mathrm{H} 32 \mathrm{C}$ & -0.037751 & 0.605256 & -0.246168 & $0.101 *$ \\
\hline $\mathrm{C} 22$ & $0.4373(5)$ & $0.6511(10)$ & $0.0162(4)$ & $0.064(2)$ \\
\hline $\mathrm{H} 22 \mathrm{~A}$ & 0.416191 & 0.732765 & -0.028357 & $0.077 *$ \\
\hline H22B & 0.517365 & 0.635850 & 0.038173 & $0.077^{*}$ \\
\hline $\mathrm{C} 27$ & $0.1312(6)$ & $0.8883(7)$ & -0.0493 & $0.0552(18)$ \\
\hline $\mathrm{H} 27 \mathrm{~A}$ & 0.101814 & 0.926492 & -0.111198 & $0.066^{*}$ \\
\hline
\end{tabular}




$\begin{array}{lllll}\text { H27B } & 0.132010 & 0.972060 & -0.009604 & 0.066^{*} \\ \text { C29 } & 0.3272(7) & 0.8968(8) & 0.1403(5) & 0.068(2) \\ \text { H29A } & 0.368891 & 0.856492 & 0.200143 & 0.102^{*} \\ \text { H29B } & 0.259425 & 0.942465 & 0.137844 & 0.102^{*} \\ \text { H29C } & 0.371164 & 0.972534 & 0.126705 & 0.102^{*} \\ \text { C19 } & 0.2927(7) & 0.3391(10) & -0.1474(5) & 0.069(2) \\ \text { H19 } & 0.270015 & 0.312455 & -0.208304 & 0.082^{*} \\ \text { C20 } & 0.3427(6) & 0.4734(12) & -0.1196(4) & 0.070(2) \\ \text { H20 } & 0.353114 & 0.539853 & -0.160608 & 0.084^{*}\end{array}$

Atomic displacement parameters $\left(A^{2}\right)$

\begin{tabular}{|c|c|c|c|c|c|c|}
\hline & $U^{11}$ & $U^{22}$ & $U^{33}$ & $U^{12}$ & $U^{13}$ & $U^{23}$ \\
\hline Ni1 & $0.01393(19)$ & $0.0171(2)$ & $0.0136(2)$ & $0.0000(2)$ & $0.00431(17)$ & $0.0017(2)$ \\
\hline $\mathrm{Ni} 2$ & $0.0158(2)$ & $0.0210(3)$ & $0.0153(2)$ & $-0.0019(2)$ & $0.00406(17)$ & $-0.0011(2)$ \\
\hline $\mathrm{Cl} 2$ & $0.0180(4)$ & $0.0431(7)$ & $0.0155(4)$ & $0.0016(4)$ & $0.0057(3)$ & $0.0037(4)$ \\
\hline $\mathrm{Cl} 3$ & $0.0178(4)$ & $0.0225(5)$ & $0.0194(5)$ & $0.0017(4)$ & $0.0034(4)$ & $-0.0031(4)$ \\
\hline Cl1 & $0.0240(5)$ & $0.0198(5)$ & $0.0244(5)$ & $-0.0048(4)$ & $0.0031(4)$ & 0.0052 (4) \\
\hline $\mathrm{Cl} 4$ & $0.0301(5)$ & $0.0180(5)$ & $0.0367(7)$ & $-0.0042(4)$ & $-0.0027(5)$ & $0.0021(5)$ \\
\hline $\mathrm{O} 1$ & $0.0275(15)$ & $0.0233(17)$ & $0.0205(16)$ & $0.0014(13)$ & $0.0077(13)$ & $-0.0051(13)$ \\
\hline N1 & $0.0159(14)$ & $0.0249(19)$ & $0.0133(16)$ & $0.0017(13)$ & $0.0054(13)$ & $0.0017(14)$ \\
\hline N3 & $0.0163(13)$ & $0.0206(17)$ & $0.0170(15)$ & $0.0001(15)$ & $0.0056(12)$ & $0.0000(16)$ \\
\hline N2 & $0.0122(13)$ & $0.0204(19)$ & $0.0200(18)$ & $0.0019(13)$ & $0.0044(13)$ & $0.0019(14)$ \\
\hline $\mathrm{O} 2$ & $0.0186(14)$ & $0.052(3)$ & $0.0312(19)$ & $-0.0003(14)$ & $0.0099(13)$ & $0.0128(17)$ \\
\hline $\mathrm{C} 8$ & $0.0184(18)$ & $0.026(2)$ & $0.020(2)$ & $0.0079(16)$ & $0.0113(16)$ & $0.0058(17)$ \\
\hline $\mathrm{C} 5$ & $0.0221(18)$ & $0.025(2)$ & $0.0146(19)$ & $0.0034(17)$ & $0.0086(15)$ & $0.0006(17)$ \\
\hline N6 & $0.0182(15)$ & $0.028(2)$ & $0.0163(17)$ & $0.0011(13)$ & $0.0055(14)$ & $-0.0007(14)$ \\
\hline N5 & $0.029(2)$ & $0.040(3)$ & $0.033(2)$ & $-0.0178(19)$ & $-0.0040(18)$ & $0.020(2)$ \\
\hline $\mathrm{C} 12$ & $0.0200(18)$ & $0.025(2)$ & $0.027(2)$ & $-0.0009(17)$ & $0.0064(17)$ & $0.0088(19)$ \\
\hline $\mathrm{C} 14$ & $0.0249(19)$ & $0.033(3)$ & $0.016(2)$ & $0.0063(18)$ & $0.0045(16)$ & $0.0044(19)$ \\
\hline $\mathrm{C} 3$ & $0.025(2)$ & $0.036(3)$ & $0.029(3)$ & $0.0125(19)$ & $0.0111(19)$ & $0.004(2)$ \\
\hline C9 & $0.032(2)$ & $0.037(3)$ & $0.018(2)$ & $0.011(2)$ & $0.0145(18)$ & $0.006(2)$ \\
\hline $\mathrm{C} 1$ & $0.0194(17)$ & $0.028(2)$ & $0.019(2)$ & $0.0006(16)$ & $0.0077(15)$ & $0.0024(17)$ \\
\hline $\mathrm{C} 30$ & $0.035(2)$ & $0.041(3)$ & $0.033(3)$ & $0.006(2)$ & $0.015(2)$ & $0.000(2)$ \\
\hline N4 & $0.0223(18)$ & $0.061(3)$ & $0.021(2)$ & $0.0157(19)$ & $0.0044(16)$ & $-0.005(2)$ \\
\hline $\mathrm{C} 2$ & $0.0149(17)$ & $0.041(3)$ & $0.028(2)$ & $0.0019(18)$ & $0.0097(17)$ & $0.007(2)$ \\
\hline $\mathrm{C} 25$ & $0.033(2)$ & $0.047(3)$ & $0.031(3)$ & $0.022(2)$ & $0.009(2)$ & $-0.001(2)$ \\
\hline $\mathrm{C} 7$ & $0.0174(16)$ & $0.025(2)$ & $0.022(2)$ & $0.0014(16)$ & $0.0105(15)$ & 0.0077 (18) \\
\hline $\mathrm{C} 4$ & $0.025(2)$ & $0.032(3)$ & $0.027(2)$ & $0.0106(18)$ & $0.0114(18)$ & $0.002(2)$ \\
\hline $\mathrm{C} 24$ & 0.029 (2) & $0.025(2)$ & $0.017(2)$ & $0.0080(18)$ & $0.0041(18)$ & $-0.0052(18)$ \\
\hline C6 & $0.0243(19)$ & $0.017(2)$ & $0.024(2)$ & $0.0046(16)$ & $0.0094(17)$ & $-0.0026(17)$ \\
\hline $\mathrm{C} 10$ & $0.030(2)$ & $0.034(3)$ & $0.024(2)$ & $0.007(2)$ & $0.0142(18)$ & $0.012(2)$ \\
\hline $\mathrm{C} 23$ & $0.048(3)$ & $0.015(2)$ & $0.032(3)$ & $-0.003(2)$ & $0.001(2)$ & $0.006(2)$ \\
\hline $\mathrm{C} 15$ & $0.0204(19)$ & $0.036(3)$ & $0.028(2)$ & $0.0014(18)$ & $0.0032(18)$ & $0.008(2)$ \\
\hline $\mathrm{C} 31$ & $0.0267(19)$ & $0.033(2)$ & $0.032(2)$ & $-0.005(2)$ & $0.0058(18)$ & 0.001 \\
\hline $\mathrm{C} 13$ & $0.0198(19)$ & $0.040(3)$ & $0.034(3)$ & $-0.001(2)$ & $0.0108(18)$ & $0.010(2)$ \\
\hline C11 & $0.026(2)$ & 0.033 & 0.035 & $0.0008(19)$ & $0.015(2)$ & $0.018(2)$ \\
\hline $\mathrm{C} 16$ & $0.033(2)$ & $0.037(3)$ & $0.024(2)$ & $0.009(2)$ & $0.0120(19)$ & $0.011(2)$ \\
\hline
\end{tabular}




\begin{tabular}{lllllll}
$\mathrm{C} 18$ & $0.059(4)$ & $0.067(4)$ & $0.032(3)$ & $0.026(4)$ & $0.003(3)$ & $-0.011(3)$ \\
$\mathrm{C} 17$ & $0.040(3)$ & $0.057(4)$ & $0.032(3)$ & $0.025(3)$ & $-0.004(2)$ & $-0.020(3)$ \\
$\mathrm{C} 26$ & $0.043(3)$ & $0.047(4)$ & $0.028(3)$ & $0.027(3)$ & $0.005(2)$ & $0.002(2)$ \\
$\mathrm{C} 21$ & $0.030(2)$ & $0.091(5)$ & $0.031(3)$ & $0.024(3)$ & $0.013(2)$ & $0.016(3)$ \\
$\mathrm{C} 28$ & $0.053(3)$ & $0.029(3)$ & $0.043(3)$ & $-0.005(3)$ & $-0.002(3)$ & $0.018(3)$ \\
$\mathrm{C} 32$ & $0.115(7)$ & $0.050(4)$ & $0.026(3)$ & $0.028(4)$ & $0.016(4)$ & $0.002(3)$ \\
$\mathrm{C} 22$ & $0.033(3)$ & $0.113(7)$ & $0.049(4)$ & $0.010(3)$ & $0.020(3)$ & $0.057(4)$ \\
$\mathrm{C} 27$ & $0.069(4)$ & $0.029(3)$ & $0.041(3)$ & $0.017(3)$ & $-0.004(3)$ & $0.007(3)$ \\
$\mathrm{C} 29$ & $0.091(5)$ & $0.027(3)$ & $0.051(4)$ & $0.000(3)$ & $-0.007(4)$ & $-0.001(3)$ \\
$\mathrm{C} 19$ & $0.093(6)$ & $0.071(5)$ & $0.055(4)$ & $0.014(4)$ & $0.044(4)$ & $-0.020(4)$ \\
$\mathrm{C} 20$ & $0.068(4)$ & $0.104(6)$ & $0.040(3)$ & $0.030(4)$ & $0.025(3)$ & $0.035(4)$ \\
\hline
\end{tabular}

Geometric parameters $\left(\AA,{ }^{\circ}\right)$

\begin{tabular}{|c|c|c|c|}
\hline $\mathrm{Ni} 1-\mathrm{Cl} 2$ & $2.4762(11)$ & $\mathrm{C} 2-\mathrm{H} 2 \mathrm{~B}$ & 0.9500 \\
\hline $\mathrm{Ni1}-\mathrm{Cl} 3$ & $2.3964(10)$ & $\mathrm{C} 25-\mathrm{H} 25 \mathrm{~A}$ & 0.9900 \\
\hline $\mathrm{Ni1}-\mathrm{Cl1}$ & $2.4408(12)$ & $\mathrm{C} 25-\mathrm{H} 25 \mathrm{~B}$ & 0.9900 \\
\hline $\mathrm{Ni1}-\mathrm{N} 1$ & $2.077(3)$ & $\mathrm{C} 25-\mathrm{C} 24$ & $1.495(6)$ \\
\hline $\mathrm{Ni} 1-\mathrm{N} 3$ & $2.041(3)$ & $\mathrm{C} 25-\mathrm{C} 26$ & $1.536(8)$ \\
\hline $\mathrm{Ni} 1-\mathrm{N} 2$ & $2.126(4)$ & $\mathrm{C} 7-\mathrm{C} 13$ & $1.545(5)$ \\
\hline $\mathrm{Ni} 2-\mathrm{Cl} 2$ & $2.4216(10)$ & $\mathrm{C} 4-\mathrm{H} 4$ & 0.9500 \\
\hline $\mathrm{Ni} 2-\mathrm{Cl} 3$ & $2.4128(12)$ & $\mathrm{C} 24-\mathrm{C} 23$ & $1.516(7)$ \\
\hline $\mathrm{Ni} 2-\mathrm{Cl} 4$ & $2.4077(14)$ & $\mathrm{C} 6-\mathrm{H} 6 \mathrm{~A}$ & 0.9900 \\
\hline $\mathrm{Ni} 2-\mathrm{N} 6$ & $2.027(3)$ & C6-H6B & 0.9900 \\
\hline $\mathrm{Ni} 2-\mathrm{N} 5$ & $2.103(4)$ & $\mathrm{C} 10-\mathrm{H} 10$ & 1.0000 \\
\hline $\mathrm{Ni} 2-\mathrm{N} 4$ & $2.095(4)$ & $\mathrm{C} 10-\mathrm{C} 11$ & $1.540(8)$ \\
\hline $\mathrm{O} 1-\mathrm{H} 1$ & $0.85(7)$ & $\mathrm{C} 23-\mathrm{C} 28$ & $1.550(8)$ \\
\hline $\mathrm{O} 1-\mathrm{N} 3$ & $1.403(5)$ & $\mathrm{C} 23-\mathrm{C} 29$ & $1.502(9)$ \\
\hline $\mathrm{N} 1-\mathrm{C} 5$ & $1.345(6)$ & $\mathrm{C} 15-\mathrm{H} 15 \mathrm{~A}$ & 0.9500 \\
\hline $\mathrm{N} 1-\mathrm{C} 1$ & $1.338(5)$ & $\mathrm{C} 15-\mathrm{H} 15 \mathrm{~B}$ & 0.9500 \\
\hline $\mathrm{N} 3-\mathrm{C} 8$ & $1.269(6)$ & $\mathrm{C} 31-\mathrm{H} 31 \mathrm{~A}$ & 0.9500 \\
\hline $\mathrm{N} 2-\mathrm{H} 2$ & $0.77(5)$ & С $31-\mathrm{H} 31 \mathrm{~B}$ & 0.9500 \\
\hline $\mathrm{N} 2-\mathrm{C} 7$ & $1.503(5)$ & $\mathrm{C} 13-\mathrm{H} 13 \mathrm{~A}$ & 0.9800 \\
\hline $\mathrm{N} 2-\mathrm{C} 6$ & $1.477(5)$ & C13-H13B & 0.9800 \\
\hline $\mathrm{O} 2-\mathrm{H} 2 \mathrm{~A}$ & $0.76(8)$ & $\mathrm{C} 13-\mathrm{H} 13 \mathrm{C}$ & 0.9800 \\
\hline $\mathrm{O} 2-\mathrm{N} 6$ & $1.385(5)$ & C11-H11A & 0.9900 \\
\hline $\mathrm{C} 8-\mathrm{C} 9$ & $1.509(6)$ & C11-H11B & 0.9900 \\
\hline $\mathrm{C} 8-\mathrm{C} 7$ & $1.513(7)$ & $\mathrm{C} 16-\mathrm{H} 16 \mathrm{~A}$ & 0.9800 \\
\hline $\mathrm{C} 5-\mathrm{C} 4$ & $1.385(6)$ & $\mathrm{C} 16-\mathrm{H} 16 \mathrm{~B}$ & 0.9800 \\
\hline $\mathrm{C} 5-\mathrm{C} 6$ & $1.508(6)$ & $\mathrm{C} 16-\mathrm{H} 16 \mathrm{C}$ & 0.9800 \\
\hline $\mathrm{N} 6-\mathrm{C} 24$ & $1.269(6)$ & C18-H18 & 0.9500 \\
\hline N5-H5 & $0.93(7)$ & $\mathrm{C} 18-\mathrm{C} 17$ & $1.370(8)$ \\
\hline $\mathrm{N} 5-\mathrm{C} 23$ & $1.517(7)$ & $\mathrm{C} 18-\mathrm{C} 19$ & $1.318(12)$ \\
\hline $\mathrm{N} 5-\mathrm{C} 22$ & $1.524(9)$ & C17-H17 & 0.9500 \\
\hline $\mathrm{C} 12-\mathrm{H} 12 \mathrm{~A}$ & 0.9900 & $\mathrm{C} 26-\mathrm{H} 26$ & 1.0000 \\
\hline $\mathrm{C} 12-\mathrm{H} 12 \mathrm{~B}$ & 0.9900 & $\mathrm{C} 26-\mathrm{C} 27$ & $1.532(10)$ \\
\hline $\mathrm{C} 12-\mathrm{C} 7$ & $1.555(6)$ & $\mathrm{C} 21-\mathrm{C} 22$ & $1.457(12)$ \\
\hline $\mathrm{C} 12-\mathrm{C} 11$ & $1.528(7)$ & $\mathrm{C} 21-\mathrm{C} 20$ & $1.465(10)$ \\
\hline
\end{tabular}




\begin{tabular}{|c|c|c|c|}
\hline $\mathrm{C} 14-\mathrm{C} 10$ & $1.523(6)$ & $\mathrm{C} 28-\mathrm{H} 28 \mathrm{~A}$ & 0.9900 \\
\hline $\mathrm{C} 14-\mathrm{C} 15$ & $1.326(7)$ & $\mathrm{C} 28-\mathrm{H} 28 \mathrm{~B}$ & 0.9900 \\
\hline $\mathrm{C} 14-\mathrm{C} 16$ & $1.506(7)$ & $\mathrm{C} 28-\mathrm{C} 27$ & $1.525(9)$ \\
\hline $\mathrm{C} 3-\mathrm{H} 3$ & 0.9500 & $\mathrm{C} 32-\mathrm{H} 32 \mathrm{~A}$ & 0.9800 \\
\hline $\mathrm{C} 3-\mathrm{C} 2$ & $1.373(8)$ & $\mathrm{C} 32-\mathrm{H} 32 \mathrm{~B}$ & 0.9800 \\
\hline $\mathrm{C} 3-\mathrm{C} 4$ & $1.384(7)$ & $\mathrm{C} 32-\mathrm{H} 32 \mathrm{C}$ & 0.9800 \\
\hline C9-H9A & 0.9900 & $\mathrm{C} 22-\mathrm{H} 22 \mathrm{~A}$ & 0.9900 \\
\hline C9-H9B & 0.9900 & $\mathrm{C} 22-\mathrm{H} 22 \mathrm{~B}$ & 0.9900 \\
\hline $\mathrm{C} 9-\mathrm{C} 10$ & $1.545(7)$ & $\mathrm{C} 27-\mathrm{H} 27 \mathrm{~A}$ & 0.9900 \\
\hline $\mathrm{C} 1-\mathrm{H} 1 \mathrm{~A}$ & 0.9500 & $\mathrm{C} 27-\mathrm{H} 27 \mathrm{~B}$ & 0.9900 \\
\hline $\mathrm{C} 1-\mathrm{C} 2$ & $1.396(6)$ & $\mathrm{C} 29-\mathrm{H} 29 \mathrm{~A}$ & 0.9800 \\
\hline $\mathrm{C} 30-\mathrm{C} 31$ & $1.318(8)$ & $\mathrm{C} 29-\mathrm{H} 29 \mathrm{~B}$ & 0.9800 \\
\hline $\mathrm{C} 30-\mathrm{C} 26$ & $1.521(8)$ & $\mathrm{C} 29-\mathrm{H} 29 \mathrm{C}$ & 0.9800 \\
\hline $\mathrm{C} 30-\mathrm{C} 32$ & $1.503(8)$ & C19-H19 & 0.9500 \\
\hline $\mathrm{N} 4-\mathrm{C} 17$ & $1.359(8)$ & $\mathrm{C} 19-\mathrm{C} 20$ & $1.360(13)$ \\
\hline $\mathrm{N} 4-\mathrm{C} 21$ & $1.314(8)$ & $\mathrm{C} 20-\mathrm{H} 20$ & 0.9500 \\
\hline $\mathrm{Cl} 3-\mathrm{Ni} 1-\mathrm{Cl} 2$ & $84.13(4)$ & $\mathrm{C} 8-\mathrm{C} 7-\mathrm{C} 13$ & $108.0(4)$ \\
\hline $\mathrm{Cl} 3-\mathrm{Ni} 1-\mathrm{Cl} 1$ & $91.99(4)$ & $\mathrm{C} 13-\mathrm{C} 7-\mathrm{C} 12$ & $110.9(4)$ \\
\hline $\mathrm{Cl} 1-\mathrm{Ni} 1-\mathrm{Cl} 2$ & $100.61(4)$ & $\mathrm{C} 5-\mathrm{C} 4-\mathrm{H} 4$ & 120.7 \\
\hline $\mathrm{N} 1-\mathrm{Ni} 1-\mathrm{Cl} 2$ & $88.69(10)$ & $\mathrm{C} 3-\mathrm{C} 4-\mathrm{C} 5$ & $118.5(5)$ \\
\hline $\mathrm{N} 1-\mathrm{Ni} 1-\mathrm{Cl} 3$ & $171.31(10)$ & $\mathrm{C} 3-\mathrm{C} 4-\mathrm{H} 4$ & 120.7 \\
\hline $\mathrm{N} 1-\mathrm{Ni1}-\mathrm{Cl1}$ & $94.14(11)$ & $\mathrm{N} 6-\mathrm{C} 24-\mathrm{C} 25$ & $124.3(5)$ \\
\hline $\mathrm{N} 1-\mathrm{Ni} 1-\mathrm{N} 2$ & $79.91(13)$ & $\mathrm{N} 6-\mathrm{C} 24-\mathrm{C} 23$ & $116.7(4)$ \\
\hline $\mathrm{N} 3-\mathrm{Ni} 1-\mathrm{Cl} 2$ & $170.10(12)$ & $\mathrm{C} 25-\mathrm{C} 24-\mathrm{C} 23$ & $118.8(4)$ \\
\hline $\mathrm{N} 3-\mathrm{Ni} 1-\mathrm{Cl} 3$ & $94.30(9)$ & $\mathrm{N} 2-\mathrm{C} 6-\mathrm{C} 5$ & $110.5(4)$ \\
\hline $\mathrm{N} 3-\mathrm{Ni} 1-\mathrm{Cl} 1$ & $89.21(12)$ & $\mathrm{N} 2-\mathrm{C} 6-\mathrm{H} 6 \mathrm{~A}$ & 109.6 \\
\hline N3-Ni1-N1 & $91.94(13)$ & $\mathrm{N} 2-\mathrm{C} 6-\mathrm{H} 6 \mathrm{~B}$ & 109.6 \\
\hline $\mathrm{N} 3-\mathrm{Ni} 1-\mathrm{N} 2$ & $77.38(15)$ & $\mathrm{C} 5-\mathrm{C} 6-\mathrm{H} 6 \mathrm{~A}$ & 109.6 \\
\hline $\mathrm{N} 2-\mathrm{Ni} 1-\mathrm{Cl} 2$ & $93.02(10)$ & $\mathrm{C} 5-\mathrm{C} 6-\mathrm{H} 6 \mathrm{~B}$ & 109.6 \\
\hline $\mathrm{N} 2-\mathrm{Ni} 1-\mathrm{Cl} 3$ & $95.56(9)$ & $\mathrm{H} 6 \mathrm{~A}-\mathrm{C} 6-\mathrm{H} 6 \mathrm{~B}$ & 108.1 \\
\hline $\mathrm{N} 2-\mathrm{Ni1}-\mathrm{Cl1}$ & $165.04(11)$ & $\mathrm{C} 14-\mathrm{C} 10-\mathrm{C} 9$ & $114.7(4)$ \\
\hline $\mathrm{Cl} 3-\mathrm{Ni} 2-\mathrm{Cl} 2$ & $84.97(4)$ & $\mathrm{C} 14-\mathrm{C} 10-\mathrm{H} 10$ & 106.7 \\
\hline $\mathrm{Cl} 4-\mathrm{Ni} 2-\mathrm{Cl} 2$ & $99.38(4)$ & $\mathrm{C} 14-\mathrm{C} 10-\mathrm{C} 11$ & $111.4(4)$ \\
\hline $\mathrm{Cl} 4-\mathrm{Ni} 2-\mathrm{Cl} 3$ & $93.14(5)$ & $\mathrm{C} 9-\mathrm{C} 10-\mathrm{H} 10$ & 106.7 \\
\hline $\mathrm{N} 6-\mathrm{Ni} 2-\mathrm{Cl} 2$ & $171.72(12)$ & $\mathrm{C} 11-\mathrm{C} 10-\mathrm{C} 9$ & $110.3(4)$ \\
\hline $\mathrm{N} 6-\mathrm{Ni} 2-\mathrm{Cl} 3$ & $92.13(11)$ & $\mathrm{C} 11-\mathrm{C} 10-\mathrm{H} 10$ & 106.7 \\
\hline $\mathrm{N} 6-\mathrm{Ni} 2-\mathrm{Cl} 4$ & $88.51(11)$ & $\mathrm{N} 5-\mathrm{C} 23-\mathrm{C} 28$ & $112.0(5)$ \\
\hline $\mathrm{N} 6-\mathrm{Ni} 2-\mathrm{N} 5$ & $79.29(16)$ & $\mathrm{C} 24-\mathrm{C} 23-\mathrm{N} 5$ & $109.5(4)$ \\
\hline $\mathrm{N} 6-\mathrm{Ni} 2-\mathrm{N} 4$ & $88.11(15)$ & $\mathrm{C} 24-\mathrm{C} 23-\mathrm{C} 28$ & $108.9(4)$ \\
\hline $\mathrm{N} 5-\mathrm{Ni} 2-\mathrm{Cl} 2$ & $93.15(13)$ & $\mathrm{C} 29-\mathrm{C} 23-\mathrm{N} 5$ & $104.7(5)$ \\
\hline $\mathrm{N} 5-\mathrm{Ni} 2-\mathrm{Cl} 3$ & $94.06(15)$ & $\mathrm{C} 29-\mathrm{C} 23-\mathrm{C} 24$ & $109.9(5)$ \\
\hline $\mathrm{N} 5-\mathrm{Ni} 2-\mathrm{Cl} 4$ & $166.04(12)$ & $\mathrm{C} 29-\mathrm{C} 23-\mathrm{C} 28$ & $111.8(5)$ \\
\hline $\mathrm{N} 4-\mathrm{Ni} 2-\mathrm{Cl} 2$ & $93.92(11)$ & $\mathrm{C} 14-\mathrm{C} 15-\mathrm{H} 15 \mathrm{~A}$ & 120.0 \\
\hline $\mathrm{N} 4-\mathrm{Ni} 2-\mathrm{Cl} 3$ & $173.62(15)$ & $\mathrm{C} 14-\mathrm{C} 15-\mathrm{H} 15 \mathrm{~B}$ & 120.0 \\
\hline $\mathrm{N} 4-\mathrm{Ni} 2-\mathrm{Cl} 4$ & $93.24(16)$ & $\mathrm{H} 15 \mathrm{~A}-\mathrm{C} 15-\mathrm{H} 15 \mathrm{~B}$ & 120.0 \\
\hline $\mathrm{N} 4-\mathrm{Ni} 2-\mathrm{N} 5$ & $79.7(2)$ & $\mathrm{C} 30-\mathrm{C} 31-\mathrm{H} 31 \mathrm{~A}$ & 120.0 \\
\hline $\mathrm{Ni} 2-\mathrm{Cl} 2-\mathrm{Ni} 1$ & $91.88(4)$ & $\mathrm{C} 30-\mathrm{C} 31-\mathrm{H} 31 \mathrm{~B}$ & 120.0 \\
\hline
\end{tabular}




\begin{tabular}{|c|c|c|c|}
\hline $\mathrm{Ni} 1-\mathrm{Cl} 3-\mathrm{Ni} 2$ & $94.09(4)$ & $\mathrm{H} 31 \mathrm{~A}-\mathrm{C} 31-\mathrm{H} 31 \mathrm{~B}$ & 120.0 \\
\hline $\mathrm{N} 3-\mathrm{O} 1-\mathrm{H} 1$ & $111(4)$ & $\mathrm{C} 7-\mathrm{C} 13-\mathrm{H} 13 \mathrm{~A}$ & 109.5 \\
\hline $\mathrm{C} 5-\mathrm{N} 1-\mathrm{Ni} 1$ & $113.6(3)$ & $\mathrm{C} 7-\mathrm{C} 13-\mathrm{H} 13 \mathrm{~B}$ & 109.5 \\
\hline $\mathrm{C} 1-\mathrm{N} 1-\mathrm{Ni} 1$ & $127.6(3)$ & $\mathrm{C} 7-\mathrm{C} 13-\mathrm{H} 13 \mathrm{C}$ & 109.5 \\
\hline $\mathrm{C} 1-\mathrm{N} 1-\mathrm{C} 5$ & $118.8(4)$ & $\mathrm{H} 13 \mathrm{~A}-\mathrm{C} 13-\mathrm{H} 13 \mathrm{~B}$ & 109.5 \\
\hline $\mathrm{O} 1-\mathrm{N} 3-\mathrm{Ni1}$ & $121.4(3)$ & $\mathrm{H} 13 \mathrm{~A}-\mathrm{C} 13-\mathrm{H} 13 \mathrm{C}$ & 109.5 \\
\hline $\mathrm{C} 8-\mathrm{N} 3-\mathrm{Ni} 1$ & $122.2(3)$ & $\mathrm{H} 13 \mathrm{~B}-\mathrm{C} 13-\mathrm{H} 13 \mathrm{C}$ & 109.5 \\
\hline $\mathrm{C} 8-\mathrm{N} 3-\mathrm{O} 1$ & $115.9(3)$ & $\mathrm{C} 12-\mathrm{C} 11-\mathrm{C} 10$ & $112.0(4)$ \\
\hline $\mathrm{Ni} 1-\mathrm{N} 2-\mathrm{H} 2$ & $93(4)$ & $\mathrm{C} 12-\mathrm{C} 11-\mathrm{H} 11 \mathrm{~A}$ & 109.2 \\
\hline $\mathrm{C} 7-\mathrm{N} 2-\mathrm{Ni} 1$ & $113.6(3)$ & $\mathrm{C} 12-\mathrm{C} 11-\mathrm{H} 11 \mathrm{~B}$ & 109.2 \\
\hline $\mathrm{C} 7-\mathrm{N} 2-\mathrm{H} 2$ & $116(4)$ & $\mathrm{C} 10-\mathrm{C} 11-\mathrm{H} 11 \mathrm{~A}$ & 109.2 \\
\hline $\mathrm{C} 6-\mathrm{N} 2-\mathrm{Ni} 1$ & $104.0(2)$ & $\mathrm{C} 10-\mathrm{C} 11-\mathrm{H} 11 \mathrm{~B}$ & 109.2 \\
\hline $\mathrm{C} 6-\mathrm{N} 2-\mathrm{H} 2$ & $109(4)$ & $\mathrm{H} 11 \mathrm{~A}-\mathrm{C} 11-\mathrm{H} 11 \mathrm{~B}$ & 107.9 \\
\hline $\mathrm{C} 6-\mathrm{N} 2-\mathrm{C} 7$ & $118.1(3)$ & $\mathrm{C} 14-\mathrm{C} 16-\mathrm{H} 16 \mathrm{~A}$ & 109.5 \\
\hline $\mathrm{N} 6-\mathrm{O} 2-\mathrm{H} 2 \mathrm{~A}$ & $105(5)$ & $\mathrm{C} 14-\mathrm{C} 16-\mathrm{H} 16 \mathrm{~B}$ & 109.5 \\
\hline $\mathrm{N} 3-\mathrm{C} 8-\mathrm{C} 9$ & $124.7(4)$ & $\mathrm{C} 14-\mathrm{C} 16-\mathrm{H} 16 \mathrm{C}$ & 109.5 \\
\hline $\mathrm{N} 3-\mathrm{C} 8-\mathrm{C} 7$ & $116.1(4)$ & $\mathrm{H} 16 \mathrm{~A}-\mathrm{C} 16-\mathrm{H} 16 \mathrm{~B}$ & 109.5 \\
\hline $\mathrm{C} 9-\mathrm{C} 8-\mathrm{C} 7$ & $119.2(4)$ & $\mathrm{H} 16 \mathrm{~A}-\mathrm{C} 16-\mathrm{H} 16 \mathrm{C}$ & 109.5 \\
\hline $\mathrm{N} 1-\mathrm{C} 5-\mathrm{C} 4$ & $122.3(4)$ & $\mathrm{H} 16 \mathrm{~B}-\mathrm{C} 16-\mathrm{H} 16 \mathrm{C}$ & 109.5 \\
\hline $\mathrm{N} 1-\mathrm{C} 5-\mathrm{C} 6$ & $115.1(4)$ & $\mathrm{C} 17-\mathrm{C} 18-\mathrm{H} 18$ & 121.9 \\
\hline $\mathrm{C} 4-\mathrm{C} 5-\mathrm{C} 6$ & $122.6(4)$ & $\mathrm{C} 19-\mathrm{C} 18-\mathrm{H} 18$ & 121.9 \\
\hline $\mathrm{O} 2-\mathrm{N} 6-\mathrm{Ni} 2$ & $122.4(3)$ & $\mathrm{C} 19-\mathrm{C} 18-\mathrm{C} 17$ & $116.1(8)$ \\
\hline $\mathrm{C} 24-\mathrm{N} 6-\mathrm{Ni} 2$ & $120.3(3)$ & $\mathrm{N} 4-\mathrm{C} 17-\mathrm{C} 18$ & $124.7(7)$ \\
\hline $\mathrm{C} 24-\mathrm{N} 6-\mathrm{O} 2$ & $116.7(4)$ & $\mathrm{N} 4-\mathrm{C} 17-\mathrm{H} 17$ & 117.7 \\
\hline $\mathrm{Ni} 2-\mathrm{N} 5-\mathrm{H} 5$ & $94(4)$ & $\mathrm{C} 18-\mathrm{C} 17-\mathrm{H} 17$ & 117.7 \\
\hline $\mathrm{C} 23-\mathrm{N} 5-\mathrm{Ni} 2$ & $112.0(3)$ & $\mathrm{C} 30-\mathrm{C} 26-\mathrm{C} 25$ & $114.3(5)$ \\
\hline $\mathrm{C} 23-\mathrm{N} 5-\mathrm{H} 5$ & $115(4)$ & $\mathrm{C} 30-\mathrm{C} 26-\mathrm{H} 26$ & 107.1 \\
\hline $\mathrm{C} 23-\mathrm{N} 5-\mathrm{C} 22$ & $118.6(4)$ & $\mathrm{C} 30-\mathrm{C} 26-\mathrm{C} 27$ & $112.5(5)$ \\
\hline $\mathrm{C} 22-\mathrm{N} 5-\mathrm{Ni} 2$ & $102.9(4)$ & $\mathrm{C} 25-\mathrm{C} 26-\mathrm{H} 26$ & 107.1 \\
\hline $\mathrm{C} 22-\mathrm{N} 5-\mathrm{H} 5$ & $110(4)$ & $\mathrm{C} 27-\mathrm{C} 26-\mathrm{C} 25$ & $108.5(5)$ \\
\hline $\mathrm{H} 12 \mathrm{~A}-\mathrm{C} 12-\mathrm{H} 12 \mathrm{~B}$ & 107.8 & $\mathrm{C} 27-\mathrm{C} 26-\mathrm{H} 26$ & 107.1 \\
\hline $\mathrm{C} 7-\mathrm{C} 12-\mathrm{H} 12 \mathrm{~A}$ & 109.0 & $\mathrm{~N} 4-\mathrm{C} 21-\mathrm{C} 22$ & $116.4(5)$ \\
\hline $\mathrm{C} 7-\mathrm{C} 12-\mathrm{H} 12 \mathrm{~B}$ & 109.0 & $\mathrm{~N} 4-\mathrm{C} 21-\mathrm{C} 20$ & $117.2(8)$ \\
\hline $\mathrm{C} 11-\mathrm{C} 12-\mathrm{H} 12 \mathrm{~A}$ & 109.0 & $\mathrm{C} 22-\mathrm{C} 21-\mathrm{C} 20$ & $126.3(7)$ \\
\hline $\mathrm{C} 11-\mathrm{C} 12-\mathrm{H} 12 \mathrm{~B}$ & 109.0 & $\mathrm{C} 23-\mathrm{C} 28-\mathrm{H} 28 \mathrm{~A}$ & 109.2 \\
\hline $\mathrm{C} 11-\mathrm{C} 12-\mathrm{C} 7$ & $113.0(4)$ & $\mathrm{C} 23-\mathrm{C} 28-\mathrm{H} 28 \mathrm{~B}$ & 109.2 \\
\hline $\mathrm{C} 15-\mathrm{C} 14-\mathrm{C} 10$ & $124.9(4)$ & $\mathrm{H} 28 \mathrm{~A}-\mathrm{C} 28-\mathrm{H} 28 \mathrm{~B}$ & 107.9 \\
\hline $\mathrm{C} 15-\mathrm{C} 14-\mathrm{C} 16$ & $120.2(4)$ & $\mathrm{C} 27-\mathrm{C} 28-\mathrm{C} 23$ & $112.2(6)$ \\
\hline $\mathrm{C} 16-\mathrm{C} 14-\mathrm{C} 10$ & $114.9(4)$ & $\mathrm{C} 27-\mathrm{C} 28-\mathrm{H} 28 \mathrm{~A}$ & 109.2 \\
\hline $\mathrm{C} 2-\mathrm{C} 3-\mathrm{H} 3$ & 120.1 & $\mathrm{C} 27-\mathrm{C} 28-\mathrm{H} 28 \mathrm{~B}$ & 109.2 \\
\hline $\mathrm{C} 2-\mathrm{C} 3-\mathrm{C} 4$ & $119.7(4)$ & $\mathrm{C} 30-\mathrm{C} 32-\mathrm{H} 32 \mathrm{~A}$ & 109.5 \\
\hline $\mathrm{C} 4-\mathrm{C} 3-\mathrm{H} 3$ & 120.1 & $\mathrm{C} 30-\mathrm{C} 32-\mathrm{H} 32 \mathrm{~B}$ & 109.5 \\
\hline $\mathrm{C} 8-\mathrm{C} 9-\mathrm{H} 9 \mathrm{~A}$ & 109.2 & $\mathrm{C} 30-\mathrm{C} 32-\mathrm{H} 32 \mathrm{C}$ & 109.5 \\
\hline $\mathrm{C} 8-\mathrm{C} 9-\mathrm{H} 9 \mathrm{~B}$ & 109.2 & $\mathrm{H} 32 \mathrm{~A}-\mathrm{C} 32-\mathrm{H} 32 \mathrm{~B}$ & 109.5 \\
\hline $\mathrm{C} 8-\mathrm{C} 9-\mathrm{C} 10$ & $112.2(4)$ & $\mathrm{H} 32 \mathrm{~A}-\mathrm{C} 32-\mathrm{H} 32 \mathrm{C}$ & 109.5 \\
\hline $\mathrm{H} 9 \mathrm{~A}-\mathrm{C} 9-\mathrm{H} 9 \mathrm{~B}$ & 107.9 & $\mathrm{H} 32 \mathrm{~B}-\mathrm{C} 32-\mathrm{H} 32 \mathrm{C}$ & 109.5 \\
\hline $\mathrm{C} 10-\mathrm{C} 9-\mathrm{H} 9 \mathrm{~A}$ & 109.2 & $\mathrm{~N} 5-\mathrm{C} 22-\mathrm{H} 22 \mathrm{~A}$ & 109.7 \\
\hline $\mathrm{C} 10-\mathrm{C} 9-\mathrm{H} 9 \mathrm{~B}$ & 109.2 & $\mathrm{~N} 5-\mathrm{C} 22-\mathrm{H} 22 \mathrm{~B}$ & 109.7 \\
\hline
\end{tabular}




\begin{tabular}{|c|c|c|c|}
\hline $\mathrm{N} 1-\mathrm{C} 1-\mathrm{H} 1 \mathrm{~A}$ & 119.0 & $\mathrm{C} 21-\mathrm{C} 22-\mathrm{N} 5$ & $110.0(4)$ \\
\hline $\mathrm{N} 1-\mathrm{C} 1-\mathrm{C} 2$ & $122.1(4)$ & $\mathrm{C} 21-\mathrm{C} 22-\mathrm{H} 22 \mathrm{~A}$ & 109.7 \\
\hline $\mathrm{C} 2-\mathrm{C} 1-\mathrm{H} 1 \mathrm{~A}$ & 119.0 & $\mathrm{C} 21-\mathrm{C} 22-\mathrm{H} 22 \mathrm{~B}$ & 109.7 \\
\hline $\mathrm{C} 31-\mathrm{C} 30-\mathrm{C} 26$ & $124.8(5)$ & $\mathrm{H} 22 \mathrm{~A}-\mathrm{C} 22-\mathrm{H} 22 \mathrm{~B}$ & 108.2 \\
\hline $\mathrm{C} 31-\mathrm{C} 30-\mathrm{C} 32$ & $120.1(5)$ & $\mathrm{C} 26-\mathrm{C} 27-\mathrm{H} 27 \mathrm{~A}$ & 109.3 \\
\hline $\mathrm{C} 32-\mathrm{C} 30-\mathrm{C} 26$ & $115.1(5)$ & $\mathrm{C} 26-\mathrm{C} 27-\mathrm{H} 27 \mathrm{~B}$ & 109.3 \\
\hline $\mathrm{C} 17-\mathrm{N} 4-\mathrm{Ni} 2$ & $126.7(4)$ & $\mathrm{C} 28-\mathrm{C} 27-\mathrm{C} 26$ & $111.5(5)$ \\
\hline $\mathrm{C} 21-\mathrm{N} 4-\mathrm{Ni} 2$ & $113.2(4)$ & $\mathrm{C} 28-\mathrm{C} 27-\mathrm{H} 27 \mathrm{~A}$ & 109.3 \\
\hline $\mathrm{C} 21-\mathrm{N} 4-\mathrm{C} 17$ & $119.9(5)$ & $\mathrm{C} 28-\mathrm{C} 27-\mathrm{H} 27 \mathrm{~B}$ & 109.3 \\
\hline $\mathrm{C} 3-\mathrm{C} 2-\mathrm{C} 1$ & $118.6(4)$ & $\mathrm{H} 27 \mathrm{~A}-\mathrm{C} 27-\mathrm{H} 27 \mathrm{~B}$ & 108.0 \\
\hline $\mathrm{C} 3-\mathrm{C} 2-\mathrm{H} 2 \mathrm{~B}$ & 120.7 & $\mathrm{C} 23-\mathrm{C} 29-\mathrm{H} 29 \mathrm{~A}$ & 109.5 \\
\hline $\mathrm{C} 1-\mathrm{C} 2-\mathrm{H} 2 \mathrm{~B}$ & 120.7 & $\mathrm{C} 23-\mathrm{C} 29-\mathrm{H} 29 \mathrm{~B}$ & 109.5 \\
\hline $\mathrm{H} 25 \mathrm{~A}-\mathrm{C} 25-\mathrm{H} 25 \mathrm{~B}$ & 107.9 & $\mathrm{C} 23-\mathrm{C} 29-\mathrm{H} 29 \mathrm{C}$ & 109.5 \\
\hline $\mathrm{C} 24-\mathrm{C} 25-\mathrm{H} 25 \mathrm{~A}$ & 109.2 & $\mathrm{H} 29 \mathrm{~A}-\mathrm{C} 29-\mathrm{H} 29 \mathrm{~B}$ & 109.5 \\
\hline $\mathrm{C} 24-\mathrm{C} 25-\mathrm{H} 25 \mathrm{~B}$ & 109.2 & $\mathrm{H} 29 \mathrm{~A}-\mathrm{C} 29-\mathrm{H} 29 \mathrm{C}$ & 109.5 \\
\hline $\mathrm{C} 24-\mathrm{C} 25-\mathrm{C} 26$ & $112.1(4)$ & $\mathrm{H} 29 \mathrm{~B}-\mathrm{C} 29-\mathrm{H} 29 \mathrm{C}$ & 109.5 \\
\hline $\mathrm{C} 26-\mathrm{C} 25-\mathrm{H} 25 \mathrm{~A}$ & 109.2 & $\mathrm{C} 18-\mathrm{C} 19-\mathrm{H} 19$ & 118.5 \\
\hline $\mathrm{C} 26-\mathrm{C} 25-\mathrm{H} 25 \mathrm{~B}$ & 109.2 & $\mathrm{C} 18-\mathrm{C} 19-\mathrm{C} 20$ & $123.0(7)$ \\
\hline $\mathrm{N} 2-\mathrm{C} 7-\mathrm{C} 8$ & $109.8(3)$ & $\mathrm{C} 20-\mathrm{C} 19-\mathrm{H} 19$ & 118.5 \\
\hline $\mathrm{N} 2-\mathrm{C} 7-\mathrm{C} 12$ & $112.5(3)$ & $\mathrm{C} 21-\mathrm{C} 20-\mathrm{H} 20$ & 120.5 \\
\hline $\mathrm{N} 2-\mathrm{C} 7-\mathrm{C} 13$ & $107.1(3)$ & $\mathrm{C} 19-\mathrm{C} 20-\mathrm{C} 21$ & $119.0(7)$ \\
\hline $\mathrm{C} 8-\mathrm{C} 7-\mathrm{C} 12$ & $108.5(3)$ & $\mathrm{C} 19-\mathrm{C} 20-\mathrm{H} 20$ & 120.5 \\
\hline $\mathrm{Ni} 1-\mathrm{N} 1-\mathrm{C} 5-\mathrm{C} 4$ & $-178.5(3)$ & $\mathrm{N} 4-\mathrm{C} 21-\mathrm{C} 20-\mathrm{C} 19$ & $2.4(9)$ \\
\hline $\mathrm{Ni} 1-\mathrm{N} 1-\mathrm{C} 5-\mathrm{C} 6$ & $2.4(4)$ & $\mathrm{C} 2-\mathrm{C} 3-\mathrm{C} 4-\mathrm{C} 5$ & $-0.9(7)$ \\
\hline $\mathrm{Ni} 1-\mathrm{N} 1-\mathrm{C} 1-\mathrm{C} 2$ & $177.8(3)$ & $\mathrm{C} 25-\mathrm{C} 24-\mathrm{C} 23-\mathrm{N} 5$ & $-168.7(4)$ \\
\hline $\mathrm{Ni1}-\mathrm{N} 3-\mathrm{C} 8-\mathrm{C} 9$ & $171.5(3)$ & $\mathrm{C} 25-\mathrm{C} 24-\mathrm{C} 23-\mathrm{C} 28$ & $-45.9(6)$ \\
\hline $\mathrm{Ni} 1-\mathrm{N} 3-\mathrm{C} 8-\mathrm{C} 7$ & $-10.6(5)$ & $\mathrm{C} 25-\mathrm{C} 24-\mathrm{C} 23-\mathrm{C} 29$ & $76.9(6)$ \\
\hline $\mathrm{Ni} 1-\mathrm{N} 2-\mathrm{C} 7-\mathrm{C} 8$ & $-7.7(4)$ & $\mathrm{C} 25-\mathrm{C} 26-\mathrm{C} 27-\mathrm{C} 28$ & $59.6(6)$ \\
\hline $\mathrm{Ni1}-\mathrm{N} 2-\mathrm{C} 7-\mathrm{C} 12$ & $-128.6(3)$ & $\mathrm{C} 7-\mathrm{N} 2-\mathrm{C} 6-\mathrm{C} 5$ & $-83.5(4)$ \\
\hline $\mathrm{Ni1}-\mathrm{N} 2-\mathrm{C} 7-\mathrm{C} 13$ & $109.3(4)$ & $\mathrm{C} 7-\mathrm{C} 8-\mathrm{C} 9-\mathrm{C} 10$ & $47.9(5)$ \\
\hline $\mathrm{Ni} 1-\mathrm{N} 2-\mathrm{C} 6-\mathrm{C} 5$ & $43.4(4)$ & $\mathrm{C} 7-\mathrm{C} 12-\mathrm{C} 11-\mathrm{C} 10$ & $-57.9(5)$ \\
\hline $\mathrm{Ni2}-\mathrm{N} 6-\mathrm{C} 24-\mathrm{C} 25$ & $173.2(4)$ & $\mathrm{C} 4-\mathrm{C} 5-\mathrm{C} 6-\mathrm{N} 2$ & $148.6(4)$ \\
\hline $\mathrm{Ni} 2-\mathrm{N} 6-\mathrm{C} 24-\mathrm{C} 23$ & $-12.5(6)$ & $\mathrm{C} 4-\mathrm{C} 3-\mathrm{C} 2-\mathrm{C} 1$ & $0.6(7)$ \\
\hline $\mathrm{Ni} 2-\mathrm{N} 5-\mathrm{C} 23-\mathrm{C} 24$ & $-13.2(5)$ & $\mathrm{C} 24-\mathrm{C} 25-\mathrm{C} 26-\mathrm{C} 30$ & $73.6(6)$ \\
\hline $\mathrm{Ni} 2-\mathrm{N} 5-\mathrm{C} 23-\mathrm{C} 28$ & $-134.2(4)$ & $\mathrm{C} 24-\mathrm{C} 25-\mathrm{C} 26-\mathrm{C} 27$ & $-52.8(6)$ \\
\hline $\mathrm{Ni} 2-\mathrm{N} 5-\mathrm{C} 23-\mathrm{C} 29$ & $104.5(5)$ & $\mathrm{C} 24-\mathrm{C} 23-\mathrm{C} 28-\mathrm{C} 27$ & $49.5(6)$ \\
\hline $\mathrm{Ni} 2-\mathrm{N} 5-\mathrm{C} 22-\mathrm{C} 21$ & $44.6(5)$ & $\mathrm{C} 6-\mathrm{N} 2-\mathrm{C} 7-\mathrm{C} 8$ & $114.5(4)$ \\
\hline $\mathrm{Ni2}-\mathrm{N} 4-\mathrm{C} 17-\mathrm{C} 18$ & $174.2(4)$ & $\mathrm{C} 6-\mathrm{N} 2-\mathrm{C} 7-\mathrm{C} 12$ & $-6.5(5)$ \\
\hline $\mathrm{Ni} 2-\mathrm{N} 4-\mathrm{C} 21-\mathrm{C} 22$ & $5.4(6)$ & $\mathrm{C} 6-\mathrm{N} 2-\mathrm{C} 7-\mathrm{C} 13$ & $-128.6(4)$ \\
\hline $\mathrm{Ni} 2-\mathrm{N} 4-\mathrm{C} 21-\mathrm{C} 20$ & $-178.0(4)$ & $\mathrm{C} 6-\mathrm{C} 5-\mathrm{C} 4-\mathrm{C} 3$ & $180.0(4)$ \\
\hline $\mathrm{O} 1-\mathrm{N} 3-\mathrm{C} 8-\mathrm{C} 9$ & $-0.7(6)$ & $\mathrm{C} 23-\mathrm{N} 5-\mathrm{C} 22-\mathrm{C} 21$ & $-79.6(6)$ \\
\hline $\mathrm{O} 1-\mathrm{N} 3-\mathrm{C} 8-\mathrm{C} 7$ & $177.3(3)$ & $\mathrm{C} 23-\mathrm{C} 28-\mathrm{C} 27-\mathrm{C} 26$ & $-59.7(7)$ \\
\hline $\mathrm{N} 1-\mathrm{C} 5-\mathrm{C} 4-\mathrm{C} 3$ & $1.0(7)$ & $\mathrm{C} 15-\mathrm{C} 14-\mathrm{C} 10-\mathrm{C} 9$ & $-24.5(7)$ \\
\hline $\mathrm{N} 1-\mathrm{C} 5-\mathrm{C} 6-\mathrm{N} 2$ & $-32.3(5)$ & $\mathrm{C} 15-\mathrm{C} 14-\mathrm{C} 10-\mathrm{C} 11$ & $101.6(6)$ \\
\hline $\mathrm{N} 1-\mathrm{C} 1-\mathrm{C} 2-\mathrm{C} 3$ & $-0.2(7)$ & $\mathrm{C} 31-\mathrm{C} 30-\mathrm{C} 26-\mathrm{C} 25$ & $-6.7(8)$ \\
\hline $\mathrm{N} 3-\mathrm{C} 8-\mathrm{C} 9-\mathrm{C} 10$ & $-134.2(4)$ & $\mathrm{C} 31-\mathrm{C} 30-\mathrm{C} 26-\mathrm{C} 27$ & $117.5(6)$ \\
\hline $\mathrm{N} 3-\mathrm{C} 8-\mathrm{C} 7-\mathrm{N} 2$ & $11.5(5)$ & $\mathrm{C} 11-\mathrm{C} 12-\mathrm{C} 7-\mathrm{N} 2$ & $172.3(3)$ \\
\hline
\end{tabular}




$\begin{array}{llll}\mathrm{N} 3-\mathrm{C} 8-\mathrm{C} 7-\mathrm{C} 12 & 134.8(4) & \mathrm{C} 11-\mathrm{C} 12-\mathrm{C} 7-\mathrm{C} 8 & 50.6(4) \\ \mathrm{N} 3-\mathrm{C} 8-\mathrm{C} 7-\mathrm{C} 13 & -104.9(4) & \mathrm{C} 11-\mathrm{C} 12-\mathrm{C} 7-\mathrm{C} 13 & -67.8(5) \\ \mathrm{O} 2-\mathrm{N} 6-\mathrm{C} 24-\mathrm{C} 25 & 1.3(6) & \mathrm{C} 16-\mathrm{C} 14-\mathrm{C} 10-\mathrm{C} 9 & 158.2(4) \\ \mathrm{O} 2-\mathrm{N} 6-\mathrm{C} 24-\mathrm{C} 23 & 175.6(4) & \mathrm{C} 16-\mathrm{C} 14-\mathrm{C} 10-\mathrm{C} 11 & -75.7(5) \\ \mathrm{C} 8-\mathrm{C} 9-\mathrm{C} 10-\mathrm{C} 14 & 77.6(5) & \mathrm{C} 18-\mathrm{C} 19-\mathrm{C} 20-\mathrm{C} 21 & 1.5(12) \\ \mathrm{C} 8-\mathrm{C} 9-\mathrm{C} 10-\mathrm{C} 11 & -49.1(5) & \mathrm{C} 17-\mathrm{N} 4-\mathrm{C} 21-\mathrm{C} 22 & -179.7(5) \\ \mathrm{C} 5-\mathrm{N} 1-\mathrm{C} 1-\mathrm{C} 2 & 0.3(6) & \mathrm{C} 17-\mathrm{N} 4-\mathrm{C} 21-\mathrm{C} 20 & -3.0(7) \\ \mathrm{N} 6-\mathrm{C} 24-\mathrm{C} 23-\mathrm{N} 5 & 16.7(6) & \mathrm{C} 17-\mathrm{C} 18-\mathrm{C} 19-\mathrm{C} 20 & -4.4(11) \\ \mathrm{N} 6-\mathrm{C} 24-\mathrm{C} 23-\mathrm{C} 28 & 139.5(5) & \mathrm{C} 26-\mathrm{C} 25-\mathrm{C} 24-\mathrm{N} 6 & -136.7(5) \\ \mathrm{N} 6-\mathrm{C} 24-\mathrm{C} 23-\mathrm{C} 29 & -97.7(5) & \mathrm{C} 26-\mathrm{C} 25-\mathrm{C} 24-\mathrm{C} 23 & 49.2(7) \\ \mathrm{N} 5-\mathrm{C} 23-\mathrm{C} 28-\mathrm{C} 27 & 170.8(5) & \mathrm{C} 21-\mathrm{N} 4-\mathrm{C} 17-\mathrm{C} 18 & 0.0(8) \\ \mathrm{C} 14-\mathrm{C} 10-\mathrm{C} 11-\mathrm{C} 12 & -72.9(5) & \mathrm{C} 32-\mathrm{C} 30-\mathrm{C} 26-\mathrm{C} 25 & 174.8(6) \\ \mathrm{C} 9-\mathrm{C} 8-\mathrm{C} 7-\mathrm{N} 2 & -170.4(3) & \mathrm{C} 32-\mathrm{C} 30-\mathrm{C} 26-\mathrm{C} 27 & -60.9(7) \\ \mathrm{C} 9-\mathrm{C} 8-\mathrm{C} 7-\mathrm{C} 12 & -47.1(5) & \mathrm{C} 22-\mathrm{N} 5-\mathrm{C} 23-\mathrm{C} 24 & 106.3(6) \\ \mathrm{C} 9-\mathrm{C} 8-\mathrm{C} 7-\mathrm{C} 13 & 73.2(5) & \mathrm{C} 22-\mathrm{N} 5-\mathrm{C} 23-\mathrm{C} 28 & -14.6(6) \\ \mathrm{C} 9-\mathrm{C} 10-\mathrm{C} 11-\mathrm{C} 12 & 55.6(5) & \mathrm{C} 22-\mathrm{N} 5-\mathrm{C} 23-\mathrm{C} 29 & -135.9(6) \\ \mathrm{C} 1-\mathrm{N} 1-\mathrm{C} 5-\mathrm{C} 4 & -0.6(6) & \mathrm{C} 22-\mathrm{C} 21-\mathrm{C} 20-\mathrm{C} 19 & 178.6(6) \\ \mathrm{C} 1-\mathrm{N} 1-\mathrm{C} 5-\mathrm{C} 6 & -179.7(4) & \mathrm{C} 29-\mathrm{C} 23-\mathrm{C} 28-\mathrm{C} 27 & -72.1(7) \\ \mathrm{C} 30-\mathrm{C} 26-\mathrm{C} 27-\mathrm{C} 28 & -67.9(6) & \mathrm{C} 19-\mathrm{C} 18-\mathrm{C} 17-\mathrm{N} 4 & 3.8(9) \\ \mathrm{N} 4-\mathrm{C} 21-\mathrm{C} 22-\mathrm{N} 5 & -35.0(7) & \mathrm{C} 20-\mathrm{C} 21-\mathrm{C} 22-\mathrm{N} 5 & 148.7(6)\end{array}$

Hydrogen-bond geometry $\left(\AA,{ }^{\circ}\right)$

\begin{tabular}{lllll}
\hline$D-\mathrm{H} \cdots A$ & $D-\mathrm{H}$ & $\mathrm{H} \cdots A$ & $D \cdots A$ & $D-\mathrm{H} \cdots A$ \\
\hline $\mathrm{O} 1-\mathrm{H} 1 \cdots \mathrm{Cl} 1$ & $0.85(7)$ & $2.32(6)$ & $3.009(4)$ & $139(6)$ \\
$\mathrm{N} 2-\mathrm{H} 2 \cdots \mathrm{Cl} 4$ & $0.77(5)$ & $2.46(5)$ & $3.209(4)$ & $166(5)$ \\
$\mathrm{O} 2-\mathrm{H} 2 A \cdots \mathrm{Cl} 4$ & $0.76(8)$ & $2.31(7)$ & $2.978(4)$ & $147(7)$ \\
$\mathrm{C} 3-\mathrm{H} 3 \cdots \mathrm{O} 1^{\mathrm{i}}$ & 0.95 & 2.58 & $3.432(5)$ & 149 \\
$\mathrm{C} 1-\mathrm{H} 1 A \cdots \mathrm{Cl} 1$ & 0.95 & 2.75 & $3.369(5)$ & 124 \\
$\mathrm{C} 6-\mathrm{H} 6 A \cdots \mathrm{Cl} 2$ & 0.99 & 2.76 & $3.309(5)$ & 115 \\
$\mathrm{C} 11-\mathrm{H} 11 B \cdots \mathrm{Cl} 3^{\mathrm{ii}}$ & 0.99 & 2.64 & $3.573(5)$ & 156 \\
$\mathrm{C} 17-\mathrm{H} 17 \cdots \mathrm{Cl} 4$ & 0.95 & 2.69 & $3.327(6)$ & 125 \\
$\mathrm{C} 26-\mathrm{H} 26 \cdots \mathrm{O} 2^{\mathrm{iii}}$ & 1.00 & 2.56 & $3.489(6)$ & 154 \\
$\mathrm{C} 22-\mathrm{H} 22 B \cdots \mathrm{Cl} 2$ & 0.99 & 2.81 & $3.352(6)$ & 115 \\
$\mathrm{C} 19-\mathrm{H} 19 \cdots \mathrm{Cl} 1^{\text {iv }}$ & 0.95 & 2.64 & $3.570(7)$ & 167 \\
\hline
\end{tabular}

Symmetry codes: (i) $-x+2, y-1 / 2,-z+1$; (ii) $-x+1, y-1 / 2,-z+1$; (iii) $-x, y+1 / 2,-z$; (iv) $-x+1, y-1 / 2,-z$. 